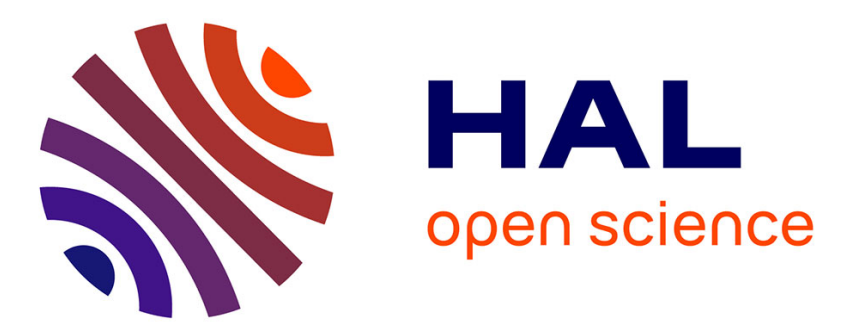

\title{
A Representation of Preferences by the Choquet Integral with Respect to a 2-Additive Capacity
}

Brice Mayag, Michel Grabisch, Christophe Labreuche

\section{To cite this version:}

Brice Mayag, Michel Grabisch, Christophe Labreuche. A Representation of Preferences by the Choquet Integral with Respect to a 2-Additive Capacity. Theory and Decision, 2011, 71 (3), pp.297-324. 10.1007/s11238-010-9198-3 . halshs-00625706

\section{HAL Id: halshs-00625706 https://shs.hal.science/halshs-00625706}

Submitted on 22 Sep 2011

HAL is a multi-disciplinary open access archive for the deposit and dissemination of scientific research documents, whether they are published or not. The documents may come from teaching and research institutions in France or abroad, or from public or private research centers.
L'archive ouverte pluridisciplinaire HAL, est destinée au dépôt et à la diffusion de documents scientifiques de niveau recherche, publiés ou non, émanant des établissements d'enseignement et de recherche français ou étrangers, des laboratoires publics ou privés. 


\section{Editorial Manager(tm) for Theory and Decision Manuscript Draft}

Manuscript Number:

Title: A representation of preferences by the Choquet integral with respect to a 2 -additive capacity Article Type: Original Research

Keywords: Capacity; Möbius transform; Choquet integral; Multiple criteria decision analysis; kmonotone function; belief function

Corresponding Author: Brice Mayag,

Corresponding Author's Institution:

First Author: Brice Mayag

Order of Authors: Brice Mayag; Michel Grabisch, Professor; Christophe Labreuche

Abstract: In the context of Multiple criteria decision analysis, we present the necessary and sufficient conditions allowing to represent an ordinal preferential information provided by the decision maker by a Choquet integral w.r.t a 2 -additive capacity. We provide also a characterization of this type of preferential information by a belief function which can be viewed as a capacity. These characterizations are based on three axioms, namely strict cycle-free preferences and some monotonicity conditions called MOPI and 2-MOPI. 
Theory and Decision manuscript No.

(will be inserted by the editor)

\title{
A representation of preferences by the Choquet integral with respect to a 2-additive capacity
}

\author{
Brice Mayag • Michel Grabisch • \\ Christophe Labreuche
}

the date of receipt and acceptance should be inserted later

\begin{abstract}
In the context of Multiple criteria decision analysis, we present the necessary and sufficient conditions allowing to represent an ordinal preferential information provided by the decision maker by a Choquet integral w.r.t a 2-additive capacity. We provide also a characterization of this type of preferential information by a belief function which can be viewed as a capacity. These characterizations are based on three axioms, namely strict cycle-free preferences and some monotonicity conditions called MOPI and 2-MOPI.
\end{abstract}

Keywords Capacity - Möbius transform - Choquet integral · Multiple criteria decision analysis $\cdot k$-monotone function $\cdot$ belief function

JEL Classification: C43; C51; C52; D63

\section{Introduction}

Since its introduction by Schmeidler [1986], the Choquet integral (see Choquet [1953]) has become a popular and flexible tool in decision making. In decision under uncertainty, as shown by the well-known Ellsberg's paradox (see Ellsberg [1961], Chateauneuf [1995]), the use of the expected utility model (see Von Neuman and Morgenstern [1944]) is limited. Therefore some non-additive

B. Mayag

Thales R \& T, Campus de Polytechnique, 1 avenue Augustin Fresnel, 91767 Palaiseau cedex,

France

E-mail: brice.mayag@thalesgroup.com

M. Grabisch

University of Paris I, Centre d'Economie de la Sorbonne, 106-112 Boulevard de l'Hôpital, 75013 Paris, France

E-mail: michel.grabisch@univ-paris1.fr

C. Labreuche

Thales R \& T

E-mail: christophe.labreuche@thalesgroup.com 
models like Choquet expected utility (see Schmeidler [1989], Chateauneuf et al. [2004]) have been proposed in order to overcome the limitations of the expected utility model. In social welfare, it generalizes the Gini index (see Weymark [1981]). In multicriteria decision making, it generalizes the weighted arithmetic mean, so far the main tool to aggregate criteria, allowing to take into account interaction between criteria (see Grabisch [1996]).

The Choquet integral is defined w.r.t. a capacity (or nonadditive monotonic measure, or fuzzy measure), and can be thought as a generalization of the expected value, the capacity playing the role of a probability measure. Supposing a finite set of criteria denoted by $N$ and having at disposal some piece of information on the preference of the decision maker, a fundamental question in practice is to determine a capacity so that the Choquet integral can represent the revealed preference of the decision maker, if such a capacity exists at all. The fact that a capacity is defined on the power set of $N$ makes the problem exponentially complex, and thus practically intractable for large sets of criteria. For this reason, many simpler models have been proposed, where the capacity is restricted to some particular subspace. These are for example symmetric capacities (depending solely on the cardinality of subsets of $N$ ), as well as their generalization called $p$-symmetric capacities (see Miranda et al. [2002]), $k$-additive capacities (see Grabisch [1997]), whose Möbius transform lives on subsets of at most $k$ elements, decomposable capacities, (see Weber [1984]) (including distorted probabilities), etc.

The concept of $k$-additive capacity seems to be of particular interest, since the value of $k$ is directly related to the complexity of the model (the number of subsets of at most $k$ elements) and it has a clear interpretation in many domains of decision making. In social welfare, the generalized Gini index proposed by Weymark [1981] corresponds in fact to a Choquet integral w.r.t. a symmetric $k$-additive capacity, and has a very natural interpretation in terms of the weight the decision maker puts on envy in the society (see Gajdos [2002], Miranda et al. [2005]). For the 2-additive case, it corresponds to a decision maker (DM) who is inequality averse in the sense that any Pigou-Dalton transfer increases his measure of welfare, wherever this transfer is applied on the income distribution. In multicriteria decision making, any interaction between two criteria can be represented and interpreted by a Choquet integral w.r.t. a 2-additive capacity, but not more complex interaction. The Choquet integral w.r.t. a 2-additive capacity is very used in many applications such that the evaluation of discomfort in sitting position (see Grabisch et al. [2002]), the construction of performance measurement systems model in a supply chain context (see Berrah and Clivillé [2007], Clivillé et al. [2007]) and complex system design (see Pignon and Labreuche [2007]).

The above development suggests that the Choquet integral w.r.t. a 2additive capacity seems to be of particular interest, and offers a good compromise between flexibility of the model and complexity. Therefore, we focus in this paper on the 2-additive model. 
Usually the DM is supposed to be able to express his preference over the set of all alternatives $X$. Because this is not feasible in most of practical situation (the cardinality of $X$ may be very large), the DM is asked to give, using pairwise comparisons, an ordinal information (a preferential information containing only a strict preference and an indifference relations) on a subset $X^{\prime} \subseteq X$ called reference set. When $X^{\prime}$ is given by the DM, it is seldom the case that the so-obtained preferential information are sufficient to specify all the parameters of the model and especially the interaction between criteria. Therefore, we propose him to reason on some fictitious alternatives which represent concrete situations. These alternatives are simple and easy to use, and are directly related to the parameters of the model. This approach is present in the MACBETH methodology for the computation of the weights for the arithmetic mean(see Bana e Costa et al. [2005]).

The use of Choquet integral requires to ensure the commensurateness between criteria i.e. one shall be able to compare any element of one point of view with any element of any other point of view. The only way to construct the utility functions with the Choquet integral uses the reference levels (see Labreuche and Grabisch [2003], Grabisch et al. [2003]). The existence of reference levels has been justified by psychologists (Simon [1956], Slovic et al. [2002], Hsee [1996]).

The set $X^{\prime}$ we use in this paper is the set of binary alternatives or binary actions denoted by $\mathcal{B}$. A binary action is an (fictitious) alternative representing a prototypical situation where on a given subset of criteria, the attributes reach a satisfactory level $\mathbf{1}$ for at most two criteria, while on the remaining ones, they are at a neutral level (neither satisfactory nor unsatisfactory) $\mathbf{0}$. The binary actions are used in many applications through the MACBETH methodology (see Bana e Costa et al. [2001, 2002], Clivillé et al. [2007]). Since these alternatives have a very simple structure and make sense for the DM, he should have no difficulty to express preference on them. Our aim is to solve the following fundamental problem: Is the preference of the decision maker representable by a Choquet integral w.r.t. a 2-additive capacity? If the answer is positive, one can extend the preference relation over the whole set $X$.

We provide necessary and sufficient conditions on the preference of the decision maker so that a Choquet integral w.r.t. a 2-additive capacity can represent the preference. The main condition, called MOPI (MOnotonicity of Preferential Information) is related to the special kind of monotonicity induced by a 2 -additive capacity. The proof of this result being constructive, we are even able to provide an example of such a capacity.

We study also the case of belief functions (see Shafer [1976]), which are particular capacities whose Möbius transform is nonnegative. They have been well studied in decision making (see, e.g., Chateauneuf and Jaffray [1989], Jaffray [1989], Jaffray and Wakker [1993]). We prove that surprisingly any ordinal information representable by a belief function is also representable by a Choquet integral w.r.t. a 2-additive capacity (but not the converse). We find the necessary and sufficient conditions so that a Choquet integral w.r.t. a 
(2-additive) belief function can represent the preference of the DM. The main condition is a strong version of the MOPI condition, called 2-MOPI. We give an interpretation of the 2-MOPI and MOPI conditions through the notion of importance of a criterion. These two conditions are very important in order to deal with inconsistencies when they occur in the ordinal information provided by the DM.

The paper is organized as follows. Section 2 sets the framework, and gives the basic material on capacities, 2-additive capacities and the Choquet integral. Section 3 introduces various concepts useful for the characterization, such as cycles, and the MOPI property. In Section 4, we introduce the 2-MOPI property for the characterization through belief functions. All proofs are given in the last section.

\section{Preliminaries}

\subsection{Notations and aim}

Let us denote by $N=\{1, \ldots, n\}$ the set of $n$ criteria and by $2^{N}$ the set of all subsets of $N$. An action (or alternative, option) $x=\left(x_{1}, \ldots, x_{n}\right)$ is identified to an element of the Cartesian product $X=X_{1} \times \cdots \times X_{n}$, where $X_{1}, \ldots, X_{n}$ represent the set of points of view or attributes. For a subset $A$ of $N$, the notation $z=\left(x_{A}, y_{N-A}\right)$ means that $z$ is defined by $z_{i}=x_{i}$ if $i \in A$, and $z_{i}=y_{i}$ otherwise. For all $i, j$ in $N$, the element $i \vee j$ denotes one of the elements $i, j$.

Our aim is to construct a preference relation over $X$. In practice (see Chateauneuf et al. [2008], Marchant [2003]) one can only ask to the DM pairwise comparisons of alternatives on a finite subset $X^{\prime}$ of $X, X^{\prime}$ having a small size. Hence we get a preference relation $\succsim_{X^{\prime}}$ on $X^{\prime}$. The question is then: how to construct a preference relation $\succsim_{X}$ on $X$, so that $\succsim_{X}$ is an extension of $\succsim_{X^{\prime}}$ ? To this end, people usually suppose that $\succsim_{X}$ is representable by an overall utility function:

$$
x \succsim_{X} \quad y \Leftrightarrow F(U(x)) \geq F(U(y))
$$

where $U(x)=\left(u_{1}\left(x_{1}\right), \ldots, u_{n}\left(x_{n}\right)\right), u_{i}: X_{i} \rightarrow \mathbb{R}$ is called a utility function, and $F: \mathbb{R}^{n} \rightarrow \mathbb{R}$ is an aggregation function. Usually, we consider a family of aggregation functions characterized by a parameter vector $\theta$ (e.g., a weight distribution over the criteria). The parameter vector $\theta$ can be deduced from the knowledge of $\succsim_{X^{\prime}}$, that is, we determine the possible values of $\theta$ for which (1) is fulfilled over $X^{\prime}$. We study the case where $F$ is the Choquet integral w.r.t. a 2-additive capacity, thus in this case the parameter vector is the 2additive capacity and $X^{\prime}$ is the set of binary actions. The aim of this paper is to give necessary and sufficient conditions on $\succsim_{X^{\prime}}$ to be represented by a Choquet integral w.r.t. a 2 -additive capacity. The model obtained in $X^{\prime}$ will be then automatically extended to $X$. 


\subsection{Capacities and the Choquet integral}

The Choquet integral w.r.t. a capacity is a well known tool in multicriteria decision making (see Schmeidler [1986], Grabisch and Labreuche [2004], Gajdos [2002], Chateauneuf [1994]). It generalizes the arithmetic mean. We consider here a particular case of the Choquet integral, where the capacity is 2-additive. We define this notion below.

\section{Definition 21}

1. A capacity on $N$ is a set function $\mu: 2^{N} \rightarrow[0,1]$ such that:

(a) $\mu(\emptyset)=0$

(b) $\mu(N)=1$

(c) $\forall A, B \in 2^{N},[A \subseteq B \Rightarrow \mu(A) \leq \mu(B)] \quad$ (monotonicity).

2. The Möbius transform (see Chateauneuf and Jaffray [1989]) of a capacity $\mu$ on $N$ is a function $m: 2^{N} \rightarrow \mathbb{R}$ defined by:

$$
m(T):=\sum_{K \subseteq T}(-1)^{|T \backslash K|} \mu(K), \forall T \in 2^{N} .
$$

When $m$ is given, it is possible to recover the original $\mu$ by the following expression:

$$
\mu(T):=\sum_{K \subseteq T} m(K), \forall T \in 2^{N} .
$$

Definition 22 A capacity $\mu$ on $N$ is said to be 2-additive if

- For all subset $T$ of $N$ such that $|T|>2, m(T)=0$;

- There exists a subset $B$ of $N$ such that $|B|=2$ and $m(B) \neq 0$.

Notations We simplify our notation for a capacity $\mu$ and its Möbius transform $m$ by using the following shorthand: $\mu_{i}:=\mu(\{i\}), \mu_{i j}:=\mu(\{i, j\}), m_{i}:=$ $m(\{i\}), m_{i j}:=m(\{i, j\})$, for all $i, j \in N, i \neq j$. Whenever we use $i$ and $j$ together, it always means that they are different.

The following important Lemma shows that a 2 -additive capacity is entirely determined by the value of the capacity on the singletons $\{i\}$ and pairs $\{i, j\}$ of $2^{N}$ :

\section{Lemma 1}

1. Let $\mu$ be a 2-additive capacity on $N$. We have

$$
\mu(K)=\sum_{\{i, j\} \subseteq K} \mu_{i j}-(|K|-2) \sum_{i \in K} \mu_{i}, \forall K \subseteq N,|K| \geq 2 .
$$

2. If the coefficients $\mu_{i}$ and $\mu_{i j}$ are given for all $i, j \in N$, then the necessary and sufficient conditions that $\mu$ is a 2-additive capacity are:

$$
\sum_{\{i, j\} \subseteq N} \mu_{i j}-(n-2) \sum_{i \in N} \mu_{i}=1
$$




$$
\begin{gathered}
\mu_{i} \geq 0, \forall i \in N \\
\sum_{i \in A \backslash\{k\}}\left(\mu_{i k}-\mu_{i}\right) \geq(|A|-2) \mu_{k}, \forall A \subseteq N,|A| \geq 2, \forall k \in A .
\end{gathered}
$$

Proof See Grabisch [1997].

For an alternative $x:=\left(x_{1}, \ldots, x_{n}\right) \in X$, the expression of the Choquet integral w.r.t. a capacity $\mu$ is given by:

$$
C_{\mu}(U(x)):=\sum_{i=1}^{n}\left(u_{\tau(i)}\left(x_{\tau(i)}\right)-u_{\tau(i-1)}\left(x_{\tau(i-1)}\right)\right) \mu(\{\tau(i), \ldots, \tau(n)\})
$$

where $\tau$ is a permutation on $N$ such that $u_{\tau(1)}\left(x_{\tau(1)}\right) \leq u_{\tau(2)}\left(x_{\tau(2)}\right) \leq \cdots \leq$ $u_{\tau(n-1)}\left(x_{\tau(n-1)}\right) \leq u_{\tau(n)}\left(x_{\tau(n)}\right)$, and $u_{\tau(0)}\left(x_{\tau(0)}\right):=0$.

A Choquet integral w.r.t. a 2-additive capacity $\mu$ is called for short a 2additive Choquet integral. Given an alternative $x:=\left(x_{1}, \ldots, x_{n}\right) \in X$, the 2-additive Choquet integral can be written also as follows (see Grabisch and Labreuche [2008]):

$$
C_{\mu}(U(x))=\sum_{i=1}^{n} v_{i} u_{i}\left(x_{i}\right)-\frac{1}{2} \sum_{\{i, j\} \subseteq N} I_{i j}\left|u_{i}\left(x_{i}\right)-u_{j}\left(x_{j}\right)\right|
$$

where $v_{i}=\sum_{K \subseteq N \backslash i} \frac{(n-|K|-1) !|K| !}{n !}(\mu(K \cup i)-\mu(K))$ is the importance of criterion $i$ corresponding to the Shapley value of $\mu$ (see Shapley [1953]) and $I_{i j}=\mu_{i j}-\mu_{i}-\mu_{j}$ is the interaction index between the two criteria $i$ and $j$ (see Grabisch [1997], Murofushi and Soneda [1993]).

\subsection{Binary actions and relations}

We assume that the DM is able to identify for each criterion $i$ two reference levels:

1. A reference level $\mathbf{1}_{i}$ in $X_{i}$ which he considers as good and completely satisfying if he could obtain it on criterion $i$, even if more attractive elements could exist. This special element corresponds to the satisficing level in the theory of bounded rationality of Simon [1956].

2. A reference level $\mathbf{0}_{i}$ in $X_{i}$ which he considers neutral on $i$. The neutral level is an element which is thought by the DM to be neither good nor bad, neither attractive nor repulsive relatively to his concerns with respect to the criterion $i$. The existence of this neutral level has roots in psychology (see Slovic et al. [2002]), and is used in bipolar models like Cumulative Prospect Theory (see Tversky and Kahneman [1992]). 
We set for convenience $u_{i}\left(\mathbf{1}_{i}\right)=1$ and $u_{i}\left(\mathbf{0}_{i}\right)=0$. The use of Choquet integral requires to ensure the commensurateness between criteria. Therefore the previous reference levels can be used in order to define the same scale on each criterion (see Labreuche and Grabisch [2003], Grabisch et al. [2003]). For more details about these reference levels, see Grabisch and Labreuche [2008, 2004].

We call a binary action or binary alternative, an element of the set

$$
\mathcal{B}=\left\{\mathbf{0}_{N},\left(\mathbf{1}_{i}, \mathbf{0}_{N-i}\right),\left(\mathbf{1}_{i j}, \mathbf{0}_{N-i j}\right), i, j \in N, i \neq j\right\} \subseteq X
$$

where

$-\mathbf{0}_{N}=\left(\mathbf{1}_{\emptyset}, \mathbf{0}_{N}\right)=: a_{0}$ is an action considered neutral on all criteria.

- $\left(\mathbf{1}_{i}, \mathbf{0}_{N-i}\right)=: a_{i}$ is an action considered satisfactory on criterion $i$ and neutral on the other criteria.

- $\left(\mathbf{1}_{i j}, \mathbf{0}_{N-i j}\right)=: a_{i j}$ is an action considered satisfactory on criteria $i$ and $j$ and neutral on the other criteria.

In MACBETH methodology (see Bana e Costa et al. [2005]), only two types of binary actions are used in order to determine the weights of the arithmetic mean: $a_{0}$ and $a_{i}, \forall i \in N$.

Using the Choquet integral, we get the following consequences:

1. For any capacity $\mu$,

$$
C_{\mu}\left(U\left(\left(\mathbf{1}_{A}, \mathbf{0}_{N-A}\right)\right)\right)=\mu(A), \forall A \subseteq N .
$$

2. For any 2-additive capacity, we have

$$
\begin{aligned}
C_{\mu}\left(U\left(a_{0}\right)\right) & =0 \\
C_{\mu}\left(U\left(a_{i}\right)\right) & =\mu_{i}=v_{i}-\frac{1}{2} \sum_{k \in N, k \neq i} I_{i k} \\
C_{\mu}\left(U\left(a_{i j}\right)\right) & =\mu_{i j}=v_{i}+v_{j}-\frac{1}{2} \sum_{k \in N, k \notin\{i, j\}}\left(I_{i k}+I_{j k}\right)
\end{aligned}
$$

The last two equations come from general relations between the capacity $\mu$ and interaction (see Grabisch [1997] for details). Generally the DM knows how to compare some alternatives using his knowledge of the problem, his experience, etc. These alternatives form a set of reference alternatives and allow to determine the parameters of a model (utility functions, subjective probabilities, weights,... ) in the decision process (see Marchant [2003] for more details). As shown by the previous equations (11),(12), (13) and Lemma 1, it should be sufficient to get some preferential information from the DM only on binary actions. To entirely determine the 2-additive capacity this information is expressed by the following relations:

$P=\{(x, y) \in \mathcal{B} \times \mathcal{B}:$ DM strictly prefers $x$ to $y\}, I=\{(x, y) \in \mathcal{B} \times \mathcal{B}:$ DM is indifferent between $x$ and $y\}$. The relation $P$ is irreflexive and asymmetric while $I$ is reflexive and symmetric.

Definition 23 The ordinal information on $\mathcal{B}$ is the structure $\{P, I\}$. 
Now we will suppose $P$ to be nonempty for any ordinal information $\{P, I\}$ ("non triviality axiom"). Before we end this section, let us introduce another relation $M$ which completes the ordinal information $\{P, I\}$ given by the DM and models the natural relations of monotonicity between binary actions. For $(x, y) \in\left\{\left(a_{i}, a_{0}\right), i \in N\right\} \cup\left\{\left(a_{i j}, a_{i}\right), i, j \in N, i \neq j\right\}$,

$$
x M y \text { if } \operatorname{not}(x(P \cup I) y) .
$$

The relation $M$ models the monotonicity conditions $\mu(\{i\}) \geq 0$ and $\mu(\{i, j\}) \geq$ $\mu(\{i\})$ for a capacity $\mu$.

Example 1 If we consider $N=\{1,2,3\}, \mathcal{B}=\left\{a_{o}, a_{1}, a_{2}, a_{3}, a_{12}, a_{13}, a_{23}\right\}$, $P=\left\{\left(a_{13}, a_{3}\right),\left(a_{2}, a_{3}\right),\left(a_{23}, 0\right)\right\}, I=\left\{\left(a_{12}, a_{1}\right)\right\}$, then the relation $M$ is $M=$ $\left\{\left(a_{1}, a_{0}\right),\left(a_{2}, a_{0}\right),\left(a_{3}, a_{0}\right),\left(a_{12}, a_{2}\right),\left(a_{13}, a_{1}\right),\left(a_{23}, a_{2}\right),\left(a_{23}, a_{3}\right)\right\}$.

\section{Remark 1}

For any 2-additive capacity $\mu$, by the definition of the relation $M$, we have for all $x, y \in \mathcal{B}, \quad x M y \Rightarrow C_{\mu}(U(x)) \geq C_{\mu}(U(y))$.

\section{The representation of the ordinal information by the Choquet integral}

An ordinal information $\{P, I\}$ is said to be representable by a 2-additive Choquet integral if there exists a 2 -additive capacity $\mu$ such that:

1. $\forall x, y \in \mathcal{B}, x P y \Rightarrow C_{\mu}(U(x))>C_{\mu}(U(y))$

2. $\forall x, y \in \mathcal{B}, x I y \Rightarrow C_{\mu}(U(x))=C_{\mu}(U(y))$.

Given an ordinal information $\{P, I\}$, we look for the necessary and sufficient conditions on $\mathcal{B}$ for which $\{P, I\}$ is representable by a 2 -additive Choquet integral. To do it, we need to define first the notion of strict cycle of the relation $(P \cup I \cup M)$.

\subsection{Cycle of $(P \cup I \cup M)$}

For a binary relation $\mathcal{R}$ on $\mathcal{B}$ and $x, y$ elements of $\mathcal{B},\left\{x_{1}, x_{2}, \cdots, x_{p}\right\} \subseteq \mathcal{B}$ is a path of $\mathcal{R}$ from $x$ to $y$ if $x=x_{1} \mathcal{R} x_{2} \mathcal{R} \cdots \mathcal{R} x_{p-1} \mathcal{R} x_{p}=y$. A path of $\mathcal{R}$ from $x$ to $x$ is called a cycle of $\mathcal{R}$.

- A path $\left\{x_{1}, x_{2}, \ldots, x_{p}\right\}$ of $(P \cup I \cup M)$ is said to be a strict path from $x$ to $y$ if there exists $i$ in $\{1, \ldots, p-1\}$ such that $x_{i} P x_{i+1}$. In this case, we will write $x T C_{P} y$.

- We write $x \sim y$ if there exists a nonstrict cycle of $(P \cup I \cup M)$ (hence a cycle of $(I \cup M))$ containing $x$ and $y$.

Hence we deduce the following easy proposition: 
Proposition 1 Let $\mu$ be a 2-additive capacity. Let $\{P, I\}$ be an ordinal information representable by a 2-additive Choquet integral and $x_{1}, x_{2}, \ldots, x_{p}$ be elements of $\mathcal{B}$.

If $\left(x_{1}, x_{2}, \ldots, x_{p}\right)$ is a nonstrict cycle of $(P \cup I \cup M)$ then $C_{\mu}\left(U\left(x_{1}\right)\right)=$ $C_{\mu}\left(U\left(x_{2}\right)\right)=\ldots=C_{\mu}\left(U\left(x_{p}\right)\right)$.

Proof $\left(x_{1}, x_{2}, \ldots, x_{p}\right)$ is a nonstrict cycle of $(P \cup I \cup M)$ means $x_{1}(I \cup M) x_{2}(I \cup$ $M) \ldots(I \cup M) x_{p-1}(I \cup M) x_{p}(I \cup M) x_{1}$. So using the definition of a representation of an ordinal information on $\mathcal{B}$ and the Remark 1 , we have $C_{\mu}\left(U\left(x_{1}\right)\right) \geq C_{\mu}\left(U\left(x_{2}\right)\right) \geq \ldots \geq C_{\mu}\left(U\left(x_{p}\right)\right) \geq C_{\mu}\left(U\left(x_{1}\right)\right)$.

\subsection{MOPI property and theorem of characterization}

Before defining the property we call MOPI, let us explain this new condition through a simple example:

Example 2 Suppose that the DM says : $a_{12} I a_{3}, a_{13} I a_{2}$ and $a_{1} P a_{0}$. Using the relation $M$, we have $a_{12} M a_{2} I a_{13} M a_{3} I a_{12}$. Remark that $\left(a_{12}, a_{2}, a_{13}, a_{3}, a_{12}\right)$ forms a nonstrict cycle of $(P \cup I \cup M)$. If $\{P, I\}$ is representable by a 2-additive Choquet integral $C_{\mu}$, this implies $\mu_{12}=\mu_{13}=\mu_{2}=\mu_{3}$ and $\mu_{1}>0$. However, we get a contradiction with the monotonicity constraint $\mu_{12}+\mu_{13} \geq \mu_{1}+\mu_{2}+\mu_{3}$ of a 2-additive capacity with the subset $A=\{1,2,3\}$, $k=1$ (see Equation (7) in Lemma 1).

This type of inconsistency is defined in general form by:

Definition 31 (MOPI property) Let $i, j, k \in N$, $i$ fixed.

1. We call Monotonicity of Preferential Information in $\{i, j, k\}$ w.r.t. $i$ the following property (denoted by $(\{i, j, k\}, i)-\mathbf{M O P I})$ :

$\left.\begin{array}{l}a_{i j} \sim a_{i \vee j} \\ a_{i k} \sim a_{i \vee k} \\ i \vee j \neq i \vee k\end{array}\right\} \Rightarrow\left[\operatorname{not}\left(a_{l} T C_{P} a_{0}\right), l \in\{i, j, k\} \backslash\{i \vee k, i \vee j\}\right]$

We recall that $i \vee j=i$ or $j$. If the property $(\{i, j, k\}, i)-$ MOPI is satisfied then the elements $a_{l}, l \in\{i, j, k\} \backslash\{i \vee k, i \vee j\}$ are called the neutral binary actions of $\{i, j, k\}$ w.r.t. $i$. The set of all such elements is denoted by NBA.

2. We say that $\{i, j, k\}$ satisfies the property of MOnotonicity of Preferential Information (MOPI) if $\forall l \in\{i, j, k\},(\{i, j, k\}, l)$-MOPI is satisfied.

Example 3 Let $N=\{1,2,3,4\}$ and $i=1$ fixed. The property $(\{1,2,3\}, 1)$ MOPI reads as follows:

$$
\begin{aligned}
& \left\{\begin{array}{l}
a_{12} \sim a_{2} \\
a_{13} \sim a_{1}
\end{array} \Rightarrow \operatorname{not}\left(\begin{array}{lll}
a_{3} & T C_{P} & a_{0}
\end{array}\right)\right. \\
& \text { and } \\
& \left\{\begin{array}{l}
a_{12} \sim a_{1} \\
a_{13} \sim a_{3}
\end{array} \Rightarrow \operatorname{not}\left(\begin{array}{lll}
a_{2} & T C_{P} & a_{0}
\end{array}\right)\right. \\
& \text { and } \\
& \left\{\begin{array}{l}
a_{12} \sim a_{2} \\
a_{13} \sim a_{3}
\end{array} \Rightarrow \operatorname{not}\left(\begin{array}{lll}
a_{1} & T C_{P} & a_{0}
\end{array}\right) .\right.
\end{aligned}
$$


The MOPI condition given in this paper is in fact equivalent to the more complex MOPI property presented in Mayag et al. [2008]. We give below the main result of the paper which is a theorem of characterization of consistent ordinal information $\{P, I\}$ representable by a 2-additive Choquet integral:

Theorem 1 An ordinal information $\{P, I\}$ is representable by a 2-additive Choquet integral on $\mathcal{B}$ if and only if the following conditions are satisfied:

1. $(P \cup I \cup M)$ contains no strict cycle;

2. Any subset $K$ of $N$ such that $|K|=3$ satisfies the MOPI property.

Theorem 1 characterizes the 2 -additive Choquet integral in terms of preference, and shows that only two types of inconsistencies can occur in an ordinal information given by a DM in order to represent it by a 2-additive Choquet integral. The first one is a very classical condition (no cycle in strict preferences), while the second is inherent to the special monotonicity conditions implied by a 2-additive capacity (see equations (6) and (7)). Observe however that the MOPI condition involves only subsets of three criteria, and thus remains simple. As it is shown in the proof of the theorem, this condition suffices to infer monotonicity conditions given in (7) for all subsets.

When $I=\emptyset$ in the ordinal information, the MOPI condition becomes void, hence always satisfied. Therefore, we have the following result:

\section{Corollary 1}

Any ordinal information $\{P, I\}$ on $\mathcal{B}$ such that $I=\emptyset$ is representable by a 2-additive Choquet integral if and only if $(P \cup M)$ has no strict cycle.

Furthermore any ordinal information with empty indifference for which $(P \cup M)$ has no strict cycle, can be represented by a 2-additive capacity with nonnegative interaction indices.

Is it possible to represent an ordinal information by another aggregation function instead of the 2-additive Choquet integral? If the answer is yes, can we give a similar characterization like in Theorem 1? In the next section, we will show that it is possible by using for instance belief functions.

\section{The representation of ordinal information by belief functions}

\subsection{General definitions}

Beliefs functions are one of the fundamental concepts used in the theory of evidence of Shafer [1976], and have been well studied and used in decision making (see, e.g., Jaffray [1989], Jaffray and Wakker [1993], Smets [2005]). A belief function $\mathrm{Bel}$ is defined as a capacity whose Möbius transform $m$ : $2^{N} \rightarrow \mathbb{R}$, called in this case mass distribution or basic belief assignmentis nonnegative:

$$
m(A) \geq 0, \forall A \in 2^{N}
$$

\section{Remark 2}


- The sets $A$ such that $m(A)>0$ are called the focal elements;

- If all focal elements are singletons then the mass distribution can be considered as a probability distribution;

We can have a definition of the representation of ordinal information by a belief function which is similar to the same representation by a Choquet integral (see Section 3).

Definition 41 An ordinal information $\{P, I\}$ is said to be representable by a belief function if there exists a belief function Bel such that

1. $\forall x, y \in \mathcal{B}, x P y \Rightarrow C_{B e l}(U(x))>C_{B e l}(U(y))$

2. $\forall x, y \in \mathcal{B}$, x I $y \Rightarrow C_{B e l}(U(x))=C_{B e l}(U(y))$.

By using Definition 22, a 2-additive belief function has a mass distribution $m$ characterized by:

1. $\exists i, j \in N$ such that $m(\{i, j\}) \neq 0$;

2. $\forall K \in 2^{N}$ such that $|K| \geq 3, m(K)=0$.

In the next section, Theorem 2 provides a relation between $k$-monotone functions (see Grabisch [1997], Chateauneuf and Jaffray [1989]) and belief functions, and a relation between $k$-monotone functions and the previous MOPI property.

\section{$4.2 k$-monotone functions and belief functions}

Given an integer $k \geq 2$, a set function $\mu: 2^{N} \rightarrow[0,1]$ is $k$-monotone (shorthand for: monotone of order $k$ ) if for each family $\left\{A_{1}, A_{2}, \ldots, A_{k}\right\} \subseteq 2^{N}$, we have

$$
\mu\left(\bigcup_{i=1}^{k} A_{i}\right) \geq \sum_{\emptyset \neq I \subseteq\{1, \ldots, k\}}(-1)^{|I|+1} \mu\left(\bigcap_{i \in I} A_{i}\right) .
$$

A simpler characterization of $k$-monotone functions by their Möbius inversion is given by the following proposition:

Proposition 2 Let $k$ be an integer, $k \geq 2$. Let $\mu: 2^{N} \rightarrow[0,1]$ be a set function, and $m$ be its Möbius transform. $\mu$ is $k$-monotone if and only if

$$
\sum_{A \subseteq L \subseteq B} m(L) \geq 0 \quad \forall A, B \subseteq N, A \subseteq B \text { and } 2 \leq|A| \leq k .
$$

Proof See Chateauneuf and Jaffray [1989]

It is well known that $\mu: 2^{N} \rightarrow[0,1]$ is a belief function if and only if $\mu$ is a $k$-monotone capacity for all $k \geq 2$. The following result gives another sufficient condition to obtain a belief function from a $k$-monotone and 2 -additive capacity, and relates belief function with the MOPI condition translated in terms of capacity. 


\section{Theorem 2}

Let $\mu: 2^{N} \rightarrow[0,1]$ be a set function and $k$ be an integer such that $k \geq 2$.

1. If $\mu$ is monotone, $k$-monotone and 2-additive, then $\mu$ is a belief function (precisely a 2-additive belief function);

2. If $\mu$ is monotone and $k$-monotone then $\mu$ satisfies the following property: for all $i, j, k \in N, i$ fixed

$$
\left.\begin{array}{l}
\mu_{i j}=\mu_{i \vee j} \\
\mu_{i k}=\mu_{i \vee k} \\
i \vee j \neq i \vee k
\end{array}\right\} \Rightarrow\left[\mu_{l}=0, l \in\{i, j, k\} \backslash\{i \vee k, i \vee j\}\right] .
$$

We end the paper by a characterization of ordinal information by belief functions.

\subsection{A link between belief functions and the 2-additive Choquet integral}

In this section, we give through the MOPI property (see Section 3) a link between belief functions and the 2-additive Choquet integral.

Proposition 3 Let $\{P, I\}$ be an ordinal information on $\mathcal{B}$.

If there exist $i, j, k \in N$, i fixed such that the property $(\{i, j, k\}, i)-M O P I$ is violated, then there is no belief function Bel which represents $\{P, I\}$.

Corollary 2 Every ordinal information $\{P, I\}$ on $\mathcal{B}$ representable by a belief function Bel : $2^{N} \rightarrow[0,1]$ is representable also by a 2-additive Choquet integral.

Proof Because it is obvious that an ordinal information $\{P, I\}$ for which $(P \cup I \cup M)$ contains a strict cycle is not representable by a belief function, the proof of the Corollary 2 is a consequence of the Theorem 1 and Proposition 3.

The inverse of Corollary 2 is false. If we suppose $P=\left\{\left(a_{2}, a_{0}\right)\right\}, I=\left\{\left(a_{12}, a_{1}\right)\right\}$ and $\mu$ a 2-additive capacity, we will have $\{P, I\}$ representable by a 2 -additive Choquet integral and $I_{12}=m_{12}=\mu_{12}-\mu_{1}-\mu_{2}<0$. So no belief function can represent $\{P, I\}$ in this case. Then it is interesting to look for the class of 2-additive capacities which are belief functions. In order to characterize them, we introduce a new fundamental property called 2-MOPI property:

Definition 42 An ordinal information $\{P, I\}$ satisfies the 2-MOPI property if

$$
\forall i, j \in N, i \neq j,\left[a_{i j} \sim a_{i} \Rightarrow \operatorname{not}\left(a_{j} T C_{P} a_{0}\right)\right] .
$$

The relation between the 2-MOPI property and the MOPI property is given by the following proposition:

Proposition 4 Let $\{P, I\}$ an ordinal information on $\mathcal{B}$.

$$
\{P, I\} \text { satisfies the 2-MOPI property }
$$

$\Downarrow$

$$
\forall i, j, k \in N,\{i, j, k\} \text { satisfies the MOPI property }
$$


Now we can state the main result of this section:

\section{Theorem 3}

$\{P, I\}$ is representable by a 2-additive belief function if and only if the two following conditions are satisfied:

1. $(P \cup I \cup M)$ contains no strict cycle;

2. $\{P, I\}$ satisfies the 2-MOPI property.

\subsection{Interpretation of 2-MOPI and MOPI properties}

We try to give an interpretation in terms of decision behavior of the two main conditions introduced in this paper. We assume here for clarity that the reference level $\mathbf{1}$ is a satisfactory level for the DM, while the reference level $\mathbf{0}$ is neither bad nor good (neutral).

Facing a situation where for two criteria $i$ and $j$ the DM is indifferent between the two alternatives $a_{i j}$ and $a_{i}$, the 2-MOPI property says that alternative $a_{j}$ is equivalent to alternative $a_{0}$. Hence in such a situation, the DM thinks that the criterion $j$ is not important. This is a strong condition, since it suffices that one such criterion $i$ exists to infer the "nullity" of the criterion $j$. This condition can be related to the notion of null set in generalized measure theory (see, e.g., Pap [1995]): a set $A \subseteq N$ is said to be $n u l l$ for capacity $\mu$ if $\mu(B \cup A)=\mu(B), \forall B \subseteq N \backslash A$. Taking $A=\{j\}$ and $B=\{i\}$ gives our condition 2-MOPI. Observe that for the nullity condition, $\{j\}$ would be null if for all subsets $B$ not containing $j$ we would have $\mu(B \cup j)=\mu(B)$, but the 2-MOPI condition asks to find only one singleton satisfying this equality.

The MOPI property is a weakening of the above one, and can be interpreted in a similar way. Let us consider now three criteria $i, j$ and $k$. The MOPI condition can be translated as follows (see Example 3, with $i=1, j=2$, and $k=3$ ). Suppose that $a_{i j}$ and $a_{j}$ are indifferent. As above, this would suggest that $i$ is not important for the DM, but this is relatively to $j$, or put differently,

$i$ is much less important than $j$. Suppose in addition that $a_{i k}$ is indifferent to $a_{i}$. Again, this suggests that $k$ is much less important than $i$. Since $i$ is much less important than $j$, the conclusion is that $k$ is very unimportant, hence $a_{k}$ is indifferent to $a_{0}$. This explains the first case in the MOPI condition. The second case (indifference between $a_{i k}$ and $a_{k}$, and between $a_{i j}$ and $a_{i}$ ) works exactly the same way. The third case says that $a_{i j}$ and $a_{j}$ are indifferent $(i$ is much less important than $j$ ) as well as $a_{i k}$ and $a_{k}$ ( $i$ is much less important than $k$ ). Since $i$ is much less important than both $j$ and $k$, the conclusion is that $i$ is very unimportant, so that $a_{i}$ is indifferent with $a_{0}$.

\section{Proofs}

We present here the proofs of our results given in the previous sections. Some notions will be defined here to support our purpose. 


\section{Definition 51}

- NBA $=\{x \in \mathcal{B} \mid \exists i, j, k \in N$ such that $x \in(\{i, j, k\}, i)-N B A\}$.

- $\forall x, y \in \mathcal{B}, x \mathcal{Z} y \Leftrightarrow x=a_{0}$ and $y \in \mathrm{NBA}$.

- $\forall x, y \in \mathcal{B}, x T C y \Leftrightarrow$ there exists a path of $(P \cup I \cup M)$ from $x$ to $y$.

- $\forall x, y \in \mathcal{B}, x T C^{\prime} y \Leftrightarrow$ there exists a path of $(P \cup I \cup M \cup \mathcal{Z})$ from $x$ to $y$.

- $\forall x, y \in \mathcal{B} x \sim^{\prime} y \Leftrightarrow(x=y)$ or $\left(x T C^{\prime} y\right.$ and $\left.y T C^{\prime} x\right)$.

- The map $\phi$ will indicate the bijection between $\mathcal{B}$ and $\mathcal{P}^{2}(N)=\{S \subseteq N$ : $|S| \leq 2\}$ defined by, for all $S \in \mathcal{P}^{2}(N), \phi\left(\left(\mathbf{1}_{S}, \mathbf{0}_{N-S}\right)\right):=S$.

The "zero" relation, $\mathcal{Z}$, has been introduced to indicate that if an ordinal information is representable by a 2 -additive Choquet integral $C_{\mu}$, then any neutral binary action $a_{m}$ has the same value of $\mu$ as $a_{0}$, i.e, $\mu\left(\phi\left(a_{m}\right)\right)=$ $\mu\left(\phi\left(a_{0}\right)\right)=0$. The relations $T C$ and $T C^{\prime}$ are respectively the transitive closure of $(P \cup I \cup M)$ and $(P \cup I \cup M \cup \mathcal{Z})$. We will denote by $\overline{\mathcal{B}}$ the set of all equivalence classes of the equivalence relation $\sim^{\prime}$, and by $[x]$ the equivalence class of an element $x$ of $\mathcal{B}$.

Lemma 2 Let $\mu: 2^{N} \rightarrow \mathbb{R}_{+}$be a mapping such that

$$
\sum_{j \in A \backslash\{i\}}\left(\mu_{i j}-\mu_{j}\right) \geq(|A|-2) \mu_{i}, \forall A \subseteq N,|A| \geq 2, \forall i \in A .
$$

If $\exists i, j \in N$ such that $\mu_{i}>0$ or $\mu_{i j}>0$ then we have the following results:

1. $\sum_{\{i, j\} \subseteq N} \mu_{i j}-(n-2) \sum_{i \in N} \mu_{i}>0$.

2. If we introduce

$$
\begin{aligned}
\alpha & :=\sum_{\{i, j\} \subseteq N} \mu_{i j}-(n-2) \sum_{i \in N} \mu_{i} \\
\mu_{\emptyset}^{\prime} & :=0 \\
\mu_{i}^{\prime} & :=\frac{\mu_{i}}{\alpha} \forall i \in N \\
\mu_{i j}^{\prime} & :=\frac{\mu_{i j}}{\alpha} \forall i, j \in N \\
\mu^{\prime}(K) & :=\sum_{\{i, j\} \subseteq K} \mu_{i j}^{\prime}-(|K|-2) \sum_{i \in K} \mu_{i}^{\prime}, \forall K \subseteq N,|K|>2 .
\end{aligned}
$$

Then $\mu^{\prime}$ is a 2-additive capacity on $N$.

Proof Assume that equation (17) is satisfied.

1. For $A=N$, we have

$$
\sum_{j \in N \backslash\{i\}}\left(\mu_{i j}-\mu_{j}\right) \geq(n-2) \mu_{i} \forall i \in N .
$$

Hence

$$
\sum_{i \in N} \sum_{j \in N \backslash\{i\}}\left(\mu_{i j}-\mu_{j}\right) \geq \sum_{i \in N}(n-2) \mu_{i}
$$


which means that

$$
\sum_{i \in N} \sum_{j \in N \backslash\{i\}} \mu_{i j} \geq \sum_{i \in N}(n-2) \mu_{i}+\sum_{i \in N} \sum_{j \in N \backslash\{i\}} \mu_{j} .
$$

Therefore,

$$
2 \sum_{\{i, j\} \subseteq N} \mu_{i j} \geq(n-2) \sum_{i \in N} \mu_{i}+(n-1) \sum_{i \in N} \mu_{i}
$$

- If there exists $k \in N$ with $\mu_{k}>0$, then

$$
\sum_{\{i, j\} \subseteq N} \mu_{i j} \geq \frac{(2 n-3)}{2} \sum_{i \in N} \mu_{i} \geq(n-2) \sum_{i \in N} \mu_{i}>0 .
$$

- If $\forall i \in N, \mu_{i}=0$ and there exists $k, l \in N$ with $\mu_{k l}>0$ then

$$
\sum_{\{i, j\} \subseteq N} \mu_{i j}-(n-2) \sum_{i \in N} \mu_{i} \geq \mu_{k l}>0 .
$$

2. Using Lemma 1 we easily prove that $\mu^{\prime}$ is a 2 -additive capacity.

5.1 An equivalent property to the MOPI property

Let $K \subseteq N$ and $|K|=k \geq 2$. Let $i$ be a fixed element of $K$. Let us consider the multiset or bag $K^{i}$ of $\mathcal{B}$ in which a repetition of the element $a_{i}$ is allowed.

$$
K^{i}=\{\underbrace{a_{i}, a_{i}, \ldots, a_{i}}_{(k-2) \text { times }}\} \cup\left(\bigcup_{j \in K \backslash\{i\}}\left\{a_{j}\right\}\right) .
$$

Definition 52 Let $K \subseteq N$ such that $|K|=k \geq 3$. Let $i$ be a fixed element of $K$. Let us set $K \backslash\{i\}:=\left\{j_{1}, j_{2}, \ldots, j_{k-1}\right\}$.

1. We call Monotonicity of Preferential Information-1 in $K$ w.r.t. $i$ the following property (denoted by $(K, i)-\mathbf{M O P I - 1})$ :

$\left.\begin{array}{l}a_{i j_{1}} \sim a_{l_{1}} \\ a_{i j_{2}} \sim a_{l_{2}} \\ a_{i j_{3}} \sim a_{l_{3}} \\ \vdots \\ a_{i j_{k-1}} \sim a_{l_{k-1}} \\ \left\{a_{l_{1}}, a_{l_{2}}, \ldots, a_{l_{k-1}}\right\} \subseteq K^{i}\end{array}\right\} \Rightarrow\left[\operatorname{not}\left(a_{l_{h}} T C_{P} a_{0}\right), \forall a_{l_{h}} \in K^{i} \backslash\left\{a_{l_{1}}, a_{l_{2}}, \ldots, a_{l_{k-1}}\right\}\right]$ the multiset $K^{i}$. We say that $K$ satisfies the property of Monotonicity of Preferential Information-1 (MOPI-1) if $\forall i \in K,(K, i)-M O P I-1$ is satisfied. 
2. We call Monotonicity of Preferential Information-2 in $K$ w.r.t. $i$ the following property (denoted by $(K, i)-M O P I-\mathcal{2})$ :

$$
\begin{aligned}
& a_{i j_{1}} \sim a_{i \vee j_{1}} \\
& a_{i j_{2}} \sim a_{i \vee j_{2}} \\
& a_{i j_{3}} \sim a_{i \vee j_{3}} \\
& :\left\{\Rightarrow\left[n o t\left(a_{l_{h}} T C_{P} a_{0}\right), \forall a_{l_{h}} \in K^{i} \backslash\left\{a_{i \vee j_{1}}, \ldots, a_{i \vee j_{k-1}}\right\}\right]\right. \\
& a_{i j_{k-1}} \sim a_{i \vee j_{k-1}} \\
& \left.\left\{a_{i \vee j_{1}}, \ldots, a_{i \vee j_{k-1}}\right\} \subseteq K^{i}\right\}
\end{aligned}
$$

Remark 3 Let $K \subseteq N$ such that $|K|=3$. Using the definitions of the $M O P I$ and MOPI-2 properties, it is obvious that $K$ satisfies MOPI if and only if $K$ satisfies MOPI-2.

Proposition 5 Let $K \subseteq N$ such that $|K|=k \geq 3$. Let $i$ be a fixed element of $K$. Let us set $K \backslash\{i\}:=\left\{j_{1}, j_{2}, \ldots, j_{k-1}\right\}$. If we have the following system

$$
\left\{\begin{array}{l}
a_{i j_{1}} \sim a_{l_{1}} \\
a_{i j_{2}} \sim a_{l_{2}} \\
a_{i j_{3}} \sim a_{l_{3}} \\
\vdots \\
a_{i j_{k-1}} \sim a_{l_{k-1}} \\
\left\{a_{l_{1}}, a_{l_{2}}, \ldots, a_{l_{k-1}}\right\} \subseteq K^{i}
\end{array}\right.
$$

then $\forall j \in K \backslash i,\left\{\begin{array}{l}a_{i j} \sim a_{j} \text { if } j \in\left\{l_{1}, l_{2}, \ldots, l_{k-1}\right\} \\ a_{i j} \sim a_{i} \text { otherwise }\end{array}\right.$

In other terms, the elements $\left\{a_{l_{1}}, a_{l_{2}}, \ldots, a_{l_{k-1}}\right\}$ in the MOPI-1 condition correspond to the elements $\left\{a_{i \vee j_{1}}, \ldots, a_{i \vee j_{k-1}}\right\}$ in the MOPI-2 condition using a suitable permutation.

Proof We suppose that the system (18) is satisfied and denote by $\pi$ a mapping defined by

$$
\begin{aligned}
\pi: K \backslash\{i\} & \rightarrow\left\{l_{1}, l_{2}, \ldots, l_{k-1}\right\} \\
j_{p} & \mapsto l_{p}
\end{aligned}
$$

For $j \in K \backslash\{i\}$ we write the following sequence of relations:

$$
\begin{aligned}
& a_{i j} \sim a_{\pi(j)} \\
& a_{i \pi(j)} \sim a_{\pi^{2}(j)} \\
& a_{i \pi^{2}(j)} \sim a_{\pi^{3}(j)} \\
& \vdots \\
& a_{i \pi^{l}(j)} \sim a_{\pi^{l+1}(j)} \\
& \vdots \\
& 1 \leq l \leq k-2
\end{aligned}
$$


where $\pi^{l}(j):=\underbrace{(\pi \circ \pi \circ \cdots \circ \pi)}_{l \text { times }}(j)$

1. The case $j \in\left\{l_{1}, l_{2}, \ldots, l_{k-1}\right\}$ :

Let us vary $l$ from 1 until we encounter either $\pi^{l}(j)=i$ or $\pi^{l}(j)=j$. It is always possible to do so by using the equations (18) since the $l_{h}$ 's are different when they are in $K \backslash i$ and we may have one $l_{h}=i$. In both cases we will have $\pi(j) \neq \pi^{2}(j) \neq \ldots \neq \pi^{l}(j)$.

- If we encounter $\pi^{l}(j)=i$, then $a_{i j} \sim a_{i}$ and the element $\pi^{l+1}(j)$ is not defined. Since $j \in\left\{l_{1}, l_{2}, \ldots, l_{k-1}\right\}$, there exists $k \in\left\{l_{1}, l_{2}, \ldots, l_{k-1}\right\}$ such that $a_{i k} \sim a_{j}$. Hence we have $a_{j} \precsim a_{i j} \sim a_{i} \precsim a_{i k} \sim a_{j}$ i.e. $a_{i j} \sim a_{j}$, where $\precsim:=M^{-1}$, where $M^{-1}=\{(x, y) \in \mathcal{B}:(y, x) \in M\}$.

- If we encounter $\pi^{l}(j)=j$, then we have $a_{i j} \sim a_{j}$.

2. The case $j \notin\left\{l_{1}, l_{2}, \ldots, l_{k-1}\right\}$ :

Let us vary $l$ from 1 until we encounter $\pi^{l}(j)=i$. It is always possible because $j \notin\left\{l_{1}, l_{2}, \ldots, l_{k-1}\right\} \Rightarrow i \in\left\{l_{1}, l_{2}, \ldots, l_{k-1}\right\}$ (by using the definition of $K^{i}$ ) and the equation (18) is satisfied. In this case we will have $\pi(j) \neq$ $\pi^{2}(j) \neq \ldots \neq \pi^{l}(j)$. Hence we have $a_{i j} \sim a_{i}$ and the element $\pi^{l+1}(j)$ is not defined.

Corollary 3 Let $K \subseteq N$ such that $|K|=k \geq 3$. Let $i$ be a fixed element of $K$.

(K,i)-MOPI-2 is satisfied if and only if $(K, i)-M O P I-1$ is satisfied.

Proof It is a consequence of Proposition 5.

Proposition 6 Every subset $L \subseteq N$ such that $|L|=3$ satisfies the MOPI property if and only if every subset $K$ of $N$ such that $|K|=k \geq 3$ satisfies the MOPI-1 property.

\section{Proof}

- $(\Leftarrow)$ This statement is true by using Corollary 3 and Remark 3 .

- $(\Rightarrow)$ Let us suppose that every subset $L \subseteq N$ such that $|L|=3$ satisfies the MOPI property. Let $K \subseteq N$ such that $|K|=k \geq 3$. Let $i$ be a fixed element of $K$. Let us set $K \backslash\{i\}:=\left\{j_{1}, j_{2}, \ldots, j_{k-1}\right\}$. Let us suppose

$$
\left\{\begin{array}{l}
a_{i j_{1}} \sim a_{l_{1}} \\
a_{i j_{2}} \sim a_{l_{2}} \\
a_{i j_{3}} \sim a_{l_{3}} \\
\vdots \\
a_{i j_{k-1}} \sim a_{l_{k-1}} \\
\left\{a_{l_{1}}, a_{l_{2}}, \ldots, a_{l_{k-1}}\right\} \subseteq K^{i}
\end{array}\right.
$$

and let us show that $\left[\operatorname{not}\left(a_{l_{h}} T C_{P} a_{0}\right), \forall a_{l_{h}} \in K^{i} \backslash\left\{a_{l_{1}}, a_{l_{2}}, \ldots, a_{l_{k-1}}\right\}\right]$.

Let $a_{l_{h}} \in K^{i} \backslash\left\{a_{l_{1}}, a_{l_{2}}, \ldots, a_{l_{k-1}}\right\}$.

1. The case $l_{h} \neq i$ : Since $l_{h} \in K \backslash i$ and $l_{h} \notin\left\{l_{1}, l_{2}, \ldots, l_{k-1}\right\}$, we have using Proposition 5

$$
a_{i l_{h}} \sim a_{i} .
$$


Furthermore, we have at most $k-2$ elements $a_{i}$ contained in the set $\left\{a_{l_{1}}, a_{l_{2}}, \ldots, a_{l_{k-1}}\right\}$. So there exists $j_{p} \in K \backslash i, j_{p} \neq l_{h}$ such that by Proposition 5

$$
a_{i j_{p}} \sim a_{j_{p}} .
$$

Considering the set of three elements $L=\left\{i, l_{h}, j_{p}\right\}$ where $i$ is fixed and the system formed by the two equations (19) et (20), we have $\operatorname{not}\left(a_{l_{h}} T C_{P} a_{0}\right)$ because $L=\left\{i, l_{h}, j_{p}\right\}$ satisfies the MOPI property.

2. The case $l_{h}=i$ : Because this $i$ is not in the set $\left\{l_{1}, l_{2}, \ldots, l_{k-1}\right\}$, we have by definition of $K^{i}$ at most $k-3$ elements $a_{i}$ among the elements of $\left\{a_{l_{1}}, a_{l_{2}}, \ldots, a_{l_{k-1}}\right\}$. So we can find two elements $a_{j_{p}}$ and $a_{j_{q}}$ of $\left\{a_{l_{1}}, a_{l_{2}}, \ldots, a_{l_{k-1}}\right\}$ such that $j_{p}, j_{q} \in K \backslash i$. Using Proposition 5 we get the following system:

$$
\left\{\begin{array}{l}
a_{i j_{p}} \sim a_{j_{p}} \\
a_{i j_{q}} \sim a_{j_{q}}
\end{array}\right.
$$

Hence we have $\operatorname{not}\left(a_{i} T C_{P} a_{0}\right)$ because $L=\left\{i, j_{p}, j_{q}\right\}$ satisfies the MOPI property.

\subsection{Topological sorting}

In this section we suppose the following two conditions:

1. $(P \cup I \cup M)$ contains no strict cycle.

2. Any subset $K$ of $N$ such that $|K|=k \geq 3$ is MOPI-1.

\section{Lemma 3}

1. $\forall x \in \mathcal{B} \backslash\left\{a_{o}\right\}, x T C^{\prime} a_{o}$.

2. $\forall x, y \in \mathcal{B}, x T C^{\prime} y \Leftrightarrow[x T C y]$ or $\left[\exists a_{m} \in \mathrm{NBA}\right.$ such that $\left.a_{m} T C y\right]$.

3. $\forall x \in \mathcal{B}, x \notin\left[a_{0}\right] \Rightarrow\left(\forall z, t \in[x], z \sim^{\prime} t \Leftrightarrow z \sim t\right)$.

Proof

1. The first point of the lemma is true because $\forall x \in \mathcal{B} \backslash\left\{a_{o}\right\}$, we have $x(P \cup$ $I \cup M) a_{o}$ by the definition of $(P \cup I \cup M)$.

2. If $x T C^{\prime} y$ and $\operatorname{not}(x T C y)$ then by the definition of the relations $T C, \mathcal{Z}$ and $T C^{\prime}$ (see Definition 51), there exists $a_{m} \in \mathrm{NBA}$ such that $x T C a_{0} \mathcal{Z} a_{m} T C y$ with $a_{m} \in$ NBA. Hence we easily prove the second point of the lemma.

3. Let $x \in \mathcal{B}$ such that $x \notin\left[a_{0}\right]$ and $z, t \in[x]$. If $z T C t$ then $z T C^{\prime} t$. If $z T C^{\prime} t$ and $\operatorname{not}(z T C t)$ then by using the second point of the lemma, there exists $a_{m} \in$ NBA such that $a_{m} T C t$. So, we have $z T C^{\prime} a_{0} \mathcal{Z} a_{m} T C^{\prime} t T C^{\prime} a_{0}$ i.e $t \in\left[a_{0}\right]$. This leads to a contradiction with $t \notin\left[a_{0}\right]$. Hence $z T C^{\prime} t \Leftrightarrow$ $z T C t$, and $z \sim^{\prime} t \Leftrightarrow z \sim t$ because $(P \cup I \cup M)$ contains no strict cycle.

\section{Lemma 4}

i) $\forall x, y \in \mathcal{B}$, if $x, y \in\left[a_{0}\right]$ then $\operatorname{not}(x P y)$.

ii) $\forall x \in\left[a_{0}\right], \forall y \notin\left[a_{0}\right], x T C^{\prime} y$ is false. 
Proof

i) Let $x, y \in\left[a_{0}\right]$ such that $x P y$. We have $a_{0} T C^{\prime} x$. Using the second point of Lemma 3, we have $\left[a_{0} T C x\right]$ or $\left[\exists a_{m} \in \mathrm{NBA}\right.$ such that $\left.a_{m} T C x\right]$.

If $a_{0} T C x$, then $a_{0} T C x P$ y $M a_{0}$. So, $\left(a_{0}, x, y\right)$ forms a strict cycle of $(P \cup I \cup M)$. This is impossible because $(P \cup I \cup M)$ contains no strict cycle.

If $\exists a_{m} \in$ NBA such that $a_{m} T C x$, then $a_{m} T C x P$ y $M a_{0}$. Hence, $a_{m} T C_{P} a_{0}$. This leads to a contradiction with not $\left(a_{m} T C_{P} a_{0}\right)$ because $a_{m} \in \mathrm{NBA}$.

ii) Let $x \in\left[a_{0}\right]$ and $y \notin\left[a_{0}\right]$ such that $x T C^{\prime} y$. Using the first point of the Lemma 3 and the definition of $\left[a_{0}\right]$, we have $a_{0} T C^{\prime} x T C^{\prime} y T C^{\prime} a_{0}$. Hence $y \in\left[a_{0}\right]$, a contradiction.

Let us define the following binary relation $P_{\sim}$ by: $\forall[x],[y] \in \overline{\mathcal{B}},[x] P_{\sim}[y] \Leftrightarrow$ $\exists z \in[x], \exists t \in[y]$ such that $z(P \cup M) t$. The relation $P_{\sim}$ is an asymmetric and acyclic relation because $P$ and $M$ are asymmetric and $(P \cup I \cup M)$ contains no strict cycle. Let us consider the following sets:

$$
\begin{aligned}
& \overline{\mathcal{B}}_{0}=\left\{a \in \overline{\mathcal{B}}: \forall b \in \overline{\mathcal{B}}, \operatorname{not}\left(a P_{\sim} b\right)\right\} \\
& \overline{\mathcal{B}}_{1}=\left\{a \in \overline{\mathcal{B}} \backslash \overline{\mathcal{B}}_{0}: \forall b \in \overline{\mathcal{B}} \backslash \overline{\mathcal{B}}_{0}, \operatorname{not}\left(a P_{\sim} b\right)\right\} \\
& \vdots \\
& \overline{\mathcal{B}}_{i}=\left\{a \in \overline{\mathcal{B}} \backslash\left(\overline{\mathcal{B}}_{0} \cup \ldots \cup \overline{\mathcal{B}}_{i-1}\right): \forall b \in \overline{\mathcal{B}} \backslash\left(\overline{\mathcal{B}}_{0} \cup \ldots \cup \overline{\mathcal{B}}_{i-1}\right), \operatorname{not}\left(a P_{\sim} b\right)\right\}
\end{aligned}
$$

Each set $\overline{\mathcal{B}}_{k}$ exists since $P_{\sim}$ is asymmetric and acyclic. Let $m+1$ be the number of sets $\overline{\mathcal{B}}_{i}$ which are built, $m+1$ is finite because the cardinality of $\overline{\mathcal{B}}$ is finite. The set $\left\{\overline{\mathcal{B}}_{0}, \overline{\mathcal{B}}_{1}, \ldots, \overline{\mathcal{B}}_{m}\right\}$ is called a topological sorting of $(P \cup I \cup M \cup \mathcal{Z})$, which is a classical notion often used in graph theory.

Lemma 5 The sets $\overline{\mathcal{B}}_{0}, \overline{\mathcal{B}}_{1}, \ldots, \overline{\mathcal{B}}_{m}$ form a partition of $\overline{\mathcal{B}}$.

Proof The proof comes from the construction of the sets $\overline{\mathcal{B}}_{0}, \overline{\mathcal{B}}_{1}, \ldots, \overline{\mathcal{B}}_{m}$.

Let us define for each set $\overline{\mathcal{B}}_{i}, i \in\{0, \ldots, m\}$, the set $\mathcal{B}_{i}$ by

$$
\mathcal{B}_{i}=\left\{x \in a: a \in \overline{\mathcal{B}}_{i}\right\}
$$

One may have incomparable elements in a some class $\mathcal{B}_{i}$. The sets $\mathcal{B}_{0}, \mathcal{B}_{1}, \ldots, \mathcal{B}_{m}$ form a partition of $\mathcal{B}$.

Lemma 6 Let $x, y \in \mathcal{B}$ and $i, j \in N$

1. $\mathcal{B}_{0}=\left[a_{0}\right]$.

2. If $x \sim y$ then $\exists i \in\{0, \ldots, m\}$ such that $x, y \in \mathcal{B}_{i}$.

3. $\left\{a_{i j}, a_{i}\right\} \subseteq \mathcal{B}_{s}, s \in\{1, \ldots, m\} \Rightarrow a_{i j} \sim a_{i}$.

4. $a_{i j} \in \mathcal{B}_{0} \Rightarrow\left\{a_{i}, a_{j}\right\} \subseteq \mathcal{B}_{0}$.

5. $\left[a_{i j} \in \mathcal{B}_{s}, s \in\{1, \ldots, m\}\right] \Rightarrow\left[a_{i} \in \mathcal{B}_{r}, r \leq s\right.$ and $\left.a_{j} \in \mathcal{B}_{q}, q \leq s\right]$.

6. $\left[a_{i} \in \mathcal{B}_{r}, r \in\{0, \ldots, m\}\right] \Rightarrow\left[\forall k \neq i \in N, a_{i k} \in \mathcal{B}_{s}, r \leq s\right]$.

Proof 
1. Let us prove that $\forall x \notin\left[a_{0}\right],[x] P_{\sim}\left[a_{0}\right]$ : if $x \notin\left[a_{0}\right]$, then $\forall y \in\left[a_{0}\right], x T C^{\prime} y$ and $\operatorname{not}\left(y T C^{\prime} x\right)$ by using the first point of Lemma 3 and the second point of Lemma 4. Hence $[x] P_{\sim}\left[a_{0}\right]$.

Then $\left[a_{0}\right] \in \overline{\mathcal{B}}_{0}$ and no other class $[x] \in \overline{\mathcal{B}}_{0}$ because $[x] P_{\sim}\left[a_{0}\right]$. Therefore $\mathcal{B}_{0}=\left[a_{0}\right]$.

2. If $x \sim y$ then $x \sim^{\prime} y$. Hence $[x]=[y]$ and then $\exists i \in\{0, \ldots, m\}$ such that $x, y \in \mathcal{B}_{i}$ by the definitions of the relation $\sim, \sim^{\prime}$ and the sets $\mathcal{B}_{0}, \mathcal{B}_{1}, \ldots, \mathcal{B}_{m}$.

3. If not $\left(a_{i j} \sim a_{i}\right)$ then $a_{i j}(P \cup M) a_{i}$ and not $\left(a_{i} T C a_{i j}\right)$. Using the second point of Lemma 3, we have not $\left(a_{i} T C^{\prime} a_{i j}\right)$ because $s \in\{1, \ldots, m\}$. Hence $\left[a_{i j}\right] P_{\sim}\left[a_{i}\right]$ and $\left\{a_{i j}, a_{i}\right\} \nsubseteq \mathcal{B}_{s}$, a contradiction.

Let us remark that, if $s=0$, we can have not $\left(a_{i j} \sim a_{i}\right)$ and $a_{i} T C a_{0}$ $\mathcal{Z} a_{m} T C a_{i j}$ with $a_{m} \in$ NBA, i.e $\left\{a_{i j}, a_{i}\right\} \subseteq \mathcal{B}_{0}$.

4. Property 4 is true because if $a_{i j} \in \mathcal{B}_{0}$ then $a_{0} T C^{\prime} a_{i j} T C^{\prime} a_{i} T C^{\prime} a_{0}$ and $a_{0} T C^{\prime} a_{i j} T C^{\prime} a_{j} T C^{\prime} a_{0}$. Hence $\left\{a_{i}, a_{j}\right\} \subseteq\left[a_{0}\right]=\mathcal{B}_{0}$.

5. If $a_{i j} \in \mathcal{B}_{s}, s \in\{1, \ldots, m\}$ then we have $\left[a_{i j}\right] P_{\sim}\left[a_{i}\right]$ or $\left[a_{i j}\right]=\left[a_{i}\right]$ because $(P \cup I \cup M)$ contains no strict cycle and by the definitions of $P \sim$ and $\sim$. Hence, $a_{i} \in \mathcal{B}_{r}, r \leq s$. The same argument is applied to $a_{j}$.

6. This property is the consequence of the previous properties 4 and 5 .

Lemma 7 Let $K \subseteq N$ such that $|K|=k \geq 3$ and $i$ a fixed element of $K$. Let us denote $K \backslash\{i\}=\left\{j_{1}, j_{2}, \ldots, j_{k-1}\right\}$ and $H(i)=\left\{a_{i j_{1}}, a_{i j_{2}}, \ldots, a_{i j_{k-1}}\right\}$.

If there exists $B \subseteq H(i)$ and $C \subseteq K^{i}$ such that the following two conditions are satisfied:

1. $\left\{\begin{array}{l}a_{i j_{1}} \sim a_{l_{1}} \\ a_{i j_{2}} \sim a_{l_{2}} \\ a_{i j_{3}} \sim a_{l_{3}} \\ \vdots \\ a_{i j_{p^{\prime}}} \sim a_{l_{p^{\prime}}} \\ B=\left\{a_{i j_{1}}, \ldots, a_{i j_{p^{\prime}}}\right\} \subseteq H(i) \\ C=\left\{a_{l_{1}}, \ldots, a_{l_{p^{\prime}}}\right\} \subseteq K^{i} \\ 1 \leq p^{\prime}<k-1\end{array}\right.$

2. $\forall a_{i j} \in H(i) \backslash B, \forall a_{l} \in K^{i} \backslash C, \operatorname{not}\left(a_{i j} \sim a_{l}\right)$

then

$$
\begin{cases}l_{0}<k_{0} & \text { if } k_{0} \neq 0 \\ l_{0}=k_{0} & \text { if } k_{0}=0\end{cases}
$$

where

$$
\begin{cases}k_{0}=\max \{s \in\{0,1, \ldots, m\}: & \left.\mathcal{B}_{s} \cap(H(i) \backslash B) \neq \emptyset\right\} \\ l_{0}=\max \{s \in\{0,1, \ldots, m\}: & \left.\mathcal{B}_{s} \cap\left(K^{i} \backslash C\right) \neq \emptyset\right\}\end{cases}
$$

with $m+1$ the number of sets $\overline{\mathcal{B}}_{i}$ which form the topological sorting of ( $P \cup$ $I \cup M \cup \mathcal{Z})$.

Proof $H(i) \backslash B \neq \emptyset$ since $p^{\prime}<k-1$. Hence $k_{0}$ and $l_{0}$ are well-defined. Let $a_{i j_{0}} \in \mathcal{B}_{k_{0}}$ and $a_{h_{0}} \in \mathcal{B}_{l_{0}}$. We have $a_{i j_{0}} \in H(i) \backslash B$ and $a_{h_{0}} \in K^{i} \backslash C$. 
1. Let us suppose that $l_{0}>k_{0}$.

$a_{i j_{0}} \in \mathcal{B}_{k_{0}} \Rightarrow a_{i} \in \mathcal{B}_{t}, t \leq k_{0}$ by Lemma 6 , points 4 and 5 . Hence $a_{h_{0}} \neq a_{i}$. Using Lemma 6 point $6, a_{h_{0}} \in \mathcal{B}_{l_{0}} \Rightarrow a_{i h_{0}} \in \mathcal{B}_{r}, r \geq l_{0}$. Since $r \geq l_{0}>k_{0}$, by definition of $k_{0}, a_{i h_{0}} \in B$. Hence there exists $a_{h_{1}} \in C$ such that $a_{i h_{0}} \sim$ $a_{h_{1}}$, and $a_{h_{1}} \neq a_{i}$ because $r>t$. Also, $a_{h_{1}} \neq a_{h_{0}}$ because $a_{h_{0}} \notin C$ and $a_{h_{1}} \in C$. Then $a_{i h_{1}} \in \mathcal{B}_{r_{1}}, r_{1} \geq r$, moreover $a_{i h_{1}} \in B$ since $r_{1}>k_{0}$. Since $h_{1} \neq h_{0}$, we have $a_{i h_{1}} \in \mathcal{B}_{r_{1}} \cap\left(B \backslash\left\{a_{i h_{0}}\right\}\right)$.

$a_{i h_{1}} \in B \Rightarrow \exists a_{h_{2}} \in C \backslash\left\{a_{i}, a_{h_{1}}\right\}$ such that $a_{i h_{1}} \sim a_{h_{2}}$ and $a_{i h_{2}} \in \mathcal{B}_{r_{2}} \cap$ $\left(B \backslash\left\{a_{i h_{0}}, a_{i h_{1}}\right\}\right)$ with $r_{2} \geq r_{1} \geq r \geq l_{0}>k_{0}$.

$a_{i h_{2}} \in B \Rightarrow \exists a_{h_{3}} \in C \backslash\left\{a_{i}, a_{h_{1}}, a_{h_{2}}\right\}$ such that $a_{i h_{2}} \sim a_{h_{3}}$ and $a_{i h_{3}} \in$ $\mathcal{B}_{r_{3}} \cap\left(B \backslash\left\{a_{i h_{0}}, a_{i h_{1}}, a_{i h_{2}}\right\}\right)$ with $r_{3} \geq r_{2} \geq r_{1} \geq r \geq l_{0}>k_{0}$.

We repeat the same process until exhaustion of all the elements of $C$. In the last step we will have $a_{i h_{p^{\prime}-1}} \sim a_{h_{p^{\prime}}}, a_{h_{p^{\prime}}} \in C \backslash\left\{a_{i}, a_{h_{1}}, a_{h_{2}}, \ldots, a_{h_{p^{\prime}-1}}\right\}$ and $a_{i h_{p^{\prime}}} \in \mathcal{B}_{r_{p^{\prime}}} \cap\left(B \backslash\left\{a_{i h_{0}}, a_{i h_{1}}, a_{i h_{2}}, \ldots, a_{i h_{p^{\prime}-1}}\right\}\right)$ with $r_{p^{\prime}} \geq \ldots \geq r_{2} \geq$ $r_{1} \geq r \geq l_{0}>k_{0}$. Hence we obtain $p^{\prime}+1$ elements of $B, a_{i h_{0}}, a_{i h_{1}}, \ldots, a_{i h_{p^{\prime}}}$ such that $a_{i h_{0}} \sim a_{h_{1}}, a_{i h_{1}} \sim a_{h_{2}}, \ldots, a_{i h_{p^{\prime}-1}} \sim a_{h_{p^{\prime}}}$ and $a_{i h_{p^{\prime}}} \in B$. This is a contradiction with the hypothesis $|B|=p^{\prime}$. Hence $0 \leq l_{0} \leq k_{0}$ and $l_{0}=0$ if $k_{0}=0$.

2. We suppose that $k_{0} \neq 0$ and $l_{0}=k_{0}$.

If $a_{h_{0}}=a_{i}$ then $a_{i j_{0}} \sim a_{i}$ by using Lemma 6 point 3. It is a contradiction with the hypothesis $\forall a_{i j} \in H(i) \backslash B, \forall a_{l} \in K^{i} \backslash C, \operatorname{not}\left(a_{i j} \sim a_{l}\right)$. Hence $a_{h_{0}} \neq a_{i}$ and $a_{i} \in \mathcal{B}_{t}$ with $t<k_{0}$ because $a_{i j_{0}} \in H(i) \backslash B$ and $a_{h_{0}} \in K^{i} \backslash C$ by the definition of $k_{0}$ and $l_{0}$.

$a_{h_{0}} \in \mathcal{B}_{l_{0}} \Rightarrow a_{i h_{0}} \in \mathcal{B}_{r}, r \geq l_{0}=k_{0}$.

If $r>l_{0}=k_{0}$ then $a_{i h_{0}} \in B$ by the definition of $k_{0}$. Using the reasoning of the previous point (1), we find $p^{\prime}+1$ elements of $B, a_{i h_{0}}, a_{i h_{1}}, \ldots, a_{i h_{p^{\prime}}}$ such that $a_{i h_{0}} \sim a_{h_{1}}, a_{i h_{1}} \sim a_{h_{2}}, \ldots, a_{i h_{p^{\prime}-1}} \sim a_{h_{p^{\prime}}}$ and $a_{i h_{p^{\prime}}} \in B$. It is impossible because $|B|=p^{\prime}$.

If $r=l_{0}=k_{0}$ then by using the point 3 of the Lemma 6 , we have $a_{i h_{0}} \sim a_{h_{0}}$ and $a_{i h_{0}} \in B$ because $a_{h_{0}} \notin C$. $a_{i h_{0}} \in B \Rightarrow \exists a_{h_{1}} \in C$ such that $a_{i h_{0}} \sim a_{h_{1}}$ and $a_{i h_{1}} \in \mathcal{B}_{r_{1}}$ with $r_{1} \geq r=l_{0}=k_{0}$.

If $r_{1}>r=l_{0}=k_{0}$, we apply the reasoning used in step 1 to obtain a contradiction with $|B|=p^{\prime}$.

If $r_{1}=r=l_{0}=k_{0}$ then $a_{h_{0}} \sim a_{i h_{0}} \sim a_{h_{1}} \sim a_{i h_{1}}$. Because $a_{h_{0}} \notin C$, we have necessarily $a_{i h_{1}} \in B \backslash\left\{a_{i h_{0}}\right\}$.

We repeat this process with $a_{i h_{1}}$ until the construction of $p^{\prime}+1$ elements of $B, a_{i h_{0}}, a_{i h_{1}}, \ldots, a_{i h_{p^{\prime}}}$, such that $a_{h_{0}} \sim a_{i h_{0}} \sim a_{h_{1}} \sim a_{i h_{1}} \sim a_{h_{2}} \sim a_{i h_{2}} \sim$ $a_{h_{3}} \sim a_{i h_{3}} \sim \ldots \sim a_{h_{p^{\prime}-1}} \sim a_{i h_{p^{\prime}-1}} \sim a_{h_{p^{\prime}}}$. That leads to the contradiction with $|B|=p^{\prime}$. Hence if $k_{0} \neq 0$ then $l_{0}<k_{0}$.

\subsection{Proof of Theorem 1}

Proposition 7 Let $\{P, I\}$ be an ordinal information. If the two following conditions are satisfied

1. $(P \cup I \cup M)$ contains no strict cycle 
2. Any subset $K$ of $N$ such that $|K|=k \geq 3$ is MOPI-1,

then $\{P, I\}$ is representable by a 2-additive Choquet integral.

Proof

1. Let $\left\{\mathcal{B}_{0}, \mathcal{B}_{1}, \ldots, \mathcal{B}_{m}\right\}$ be the partition of $\mathcal{B}$ built in Section 5.2. Let us define the mapping $\mu: \mathcal{B} \rightarrow \mathbb{R}$ as follows: For $i \in\{0, \ldots, m\}$,

$$
\forall x \in \mathcal{B}_{i}, \mu(\phi(x))= \begin{cases}0 & \text { if } i=0 \\ (2 n)^{i} & \text { otherwise. }\end{cases}
$$

Let $K \subseteq N$ such that $|K|=k \geq 2, i \in K$ being fixed, $K \backslash\{i\}=$ $\left\{j_{1}, j_{2}, \ldots, j_{k-1}\right\}$ and $H(i)=\left\{a_{i j_{1}}, a_{i j_{2}}, \ldots, a_{i j_{k-1}}\right\}$. We will prove that

$$
\sum_{j \in K \backslash\{i\}} \mu_{i j} \geq(k-2) \mu_{i}+\sum_{j \in K \backslash\{i\}} \mu_{j} .
$$

Using the points 4 and 5 of the Lemma 6 , the case $k=2$ is obviously satisfied. Let us suppose that $k \geq 3$.

(a) If there exists $a_{i j} \in H(i)$ and $a_{l} \in K^{i}$ such that $a_{i j} \sim a_{l}$ then there exists $p^{\prime}$ elements of $H(i)$ (without loss of the generality we are going to take the first $p^{\prime}$ elements), $B \subseteq H(i)$ and $C \subseteq K^{i}$ such that:

$$
(\mathcal{S})\left\{\begin{array}{l}
a_{i j_{1}} \sim a_{l_{1}} \\
a_{i j_{2}} \sim a_{l_{2}} \\
a_{i j_{3}} \sim a_{l_{3}} \\
\vdots \\
a_{i j_{p^{\prime}}} \sim a_{l_{p^{\prime}}} \\
B=\left\{a_{i j_{1}}, \ldots, a_{i j_{p^{\prime}}}\right\} \subseteq H(i) \\
C=\left\{a_{l_{1}}, \ldots, a_{l_{p^{\prime}}}\right\} \subseteq K^{i} \\
1 \leq p^{\prime} \leq k-1
\end{array}\right.
$$

and $\forall a_{i j} \in H(i) \backslash B, \forall a_{l} \in K^{i} \backslash C, \operatorname{not}\left(a_{i j} \sim a_{l}\right)$.

By application of the point 2 of Lemma 6 and the definition of $\mu$, the equation (22) becomes

$$
\sum_{a_{i j} \in H(i) \backslash B} \mu_{i j} \geq \sum_{a_{l} \in K^{i} \backslash C} \mu_{l}
$$

(i) If $p^{\prime}=k-1$ then we have $\forall a_{l_{k}} \in K^{i} \backslash C, a_{l_{k}} \in$ NBA because $K$ is MOPI- 1 and $a_{l_{k}} \in\left[a_{0}\right]=\mathcal{B}_{0}$ and $\mu_{l_{k}}=0$ by the definitions of $\mu,\left[a_{0}\right]$ and Lemma 6 . Hence equation (22) is satisfied.

(ii) Let us assume that $p^{\prime}<k-1$ and let

$$
\begin{aligned}
& k_{0}=\max \left\{s \in\{0,1, \ldots, m\}: \mathcal{B}_{s} \cap(H(i) \backslash B) \neq \emptyset\right\} \\
& l_{0}=\max \left\{s \in\{0,1, \ldots, m\}: \mathcal{B}_{s} \cap\left(K^{i} \backslash C\right) \neq \emptyset\right\} .
\end{aligned}
$$

Let $a_{i j_{0}} \in \mathcal{B}_{k_{0}}$ and $a_{l_{0}} \in \mathcal{B}_{l_{0}}$. 
- If $k_{0} \neq 0$ then $l_{0}<k_{0}$ (see Lemma 7$), \mu\left(\phi\left(a_{i j_{0}}\right)\right)=\mu_{i j_{0}}=$ $(2 n)^{k_{0}}$ and $\forall a_{l} \in K^{i} \backslash C, \mu\left(\phi\left(a_{l}\right)\right)=\mu_{l} \leq(2 n)^{l_{0}}$. Because

$$
l_{0}<k_{0} \Rightarrow(2 n)^{k_{0}} \geq 2 n(2 n)^{l_{0}}=(n+n)(2 n)^{l_{0}},
$$

we will have

$$
\sum_{a_{i j} \in H(i) \backslash B} \mu_{i j} \geq \mu_{i j_{0}}=(2 n)^{k_{0}} \geq(n+n)(2 n)^{l_{0}} \geq \sum_{a_{l} \in K^{i} \backslash C} \mu_{l} .
$$

Hence (23) is satisfied.

- If $k_{0}=0$ then $l_{0}=0$ (see Lemma 7) and (23) is obviously satisfied by application of Lemma 6 .

(b) Let us suppose that $\forall a_{i j} \in H(i), \forall a_{l} \in K^{i}$, not $\left(a_{i j} \sim a_{l}\right)$. Let

$$
k_{1}=\min \left\{s \in\{0,1, \ldots, m\}: a_{i j} \in \mathcal{B}_{s} \cap H(i)\right\},
$$

$a_{i j_{0}} \in \mathcal{B}_{k_{1}}, a_{i} \in \mathcal{B}_{r}$ and $a_{j_{0}} \in \mathcal{B}_{t}$. Using Lemma 6 , we have $r=t=0$ if $k_{1}=0$ and $r, t<k_{1}$ if $k_{1} \neq 0$ because we have supposed that $\operatorname{not}\left(a_{i j_{0}} \sim a_{i}\right)$ and $\operatorname{not}\left(a_{i j_{0}} \sim a_{j_{0}}\right)$. According to the definition of $\mu$, we will have $\mu\left(\phi\left(a_{i j_{0}}\right)\right)=\mu_{i j_{0}} \geq(k-2) \mu_{i}+\mu_{j_{0}}$. Furthermore $\forall j \in$ $K \backslash\left\{i, j_{0}\right\}, \mu_{i j} \geq \mu_{j}$ (see the case $k=2$ ). Hence

$$
\sum_{j \in K \backslash\{i\}} \mu_{i j}=\sum_{j \in K \backslash\left\{i, j_{0}\right\}} \mu_{i j}+\mu_{i j_{0}} \geq \sum_{j \in K \backslash\left\{i, j_{0}\right\}} \mu_{j}+(k-2) \mu_{i}+\mu_{j_{0}},
$$

and

$$
\sum_{j \in K \backslash\left\{i, j_{0}\right\}} \mu_{j}+(k-2) \mu_{i}+\mu_{j_{0}}=(k-2) \mu_{i}+\sum_{j \in K \backslash\{i\}} \mu_{j} .
$$

That means that equation (22) is satisfied.

2. Let us build a 2-additive capacity on $2^{N}$

Let $\alpha=\sum_{\{i, j\} \subseteq N} \mu_{i j}-(n-2) \sum_{i \in N} \mu_{i}$ and the mapping $\nu: 2^{N} \rightarrow[0,1]$ defined by :

$$
\left\{\begin{array}{l}
\nu_{\emptyset}=0 \\
\nu_{i}=\frac{\mu_{i}}{\alpha}, \forall i \in N \\
\nu_{i j}=\frac{\mu_{i j}}{\alpha}, \forall i, j \in N \\
\nu(K)=\sum_{\{i, j\} \subseteq K} \nu_{i j}-(|K|-2) \sum_{i \in K} \nu_{i}, \forall K \subseteq N,|K|>2 .
\end{array}\right.
$$

Because $P$ is not empty ("nontriviality axiom") and $\mu$ satisfies the equation (22) in the previous step, we have by applying Lemma 2, $\alpha>0$ and $\nu$ is a 2 - additive capacity. 
3. Let $x, y \in \mathcal{B}$.

If $x P y$ then by the definition of the sets $\mathcal{B}_{0}, \mathcal{B}_{1}, \mathcal{B}_{2}, \ldots, \mathcal{B}_{m}$, we have $x \in \mathcal{B}_{i}$ and $y \in \mathcal{B}_{j}$ with $0 \leq j<i$. Thus $C_{\nu}(U(x))=\frac{(2 n)^{i}}{\alpha}>\frac{(2 n)^{j}}{\alpha}=C_{\nu}(U(y))$ if $j>0$ or $C_{\nu}(U(x))>0=C_{\nu}(U(y))$ if $j=0$.

If $x I y$ then $x \sim y$, i.e, $x, y \in \mathcal{B}_{i}, i \in\{0, \ldots, m\}$. Thus $C_{\nu}(U(x))=$ $C_{\nu}(U(y))$.

Conclusion: $\nu$ is a 2-additive capacity such that $\{P, I\}$ is representable by a Choquet integral w.r.t. $\nu$.

Corollary 4 Let $\{P, I\}$ be an ordinal information. If the two following conditions are satisfied

1. $(P \cup I \cup M)$ contains no strict cycle

2. Any subset $K$ of $N$ such that $|K|=3$ satisfies $M O P I$,

then $\{P, I\}$ is representable by a 2-additive Choquet integral.

Proof The proof is a consequence of Propositions 6 and 7 .

Proposition 8 If an ordinal information $\{P, I\}$ is representable by a 2-additive Choquet integral then the two following conditions are satisfied:

1. $(P \cup I \cup M)$ contains no strict cycle

2. Any subset $K$ of $N$ such that $|K|=3$ satisfies $M O P I$.

Proof Let us suppose that $\mu$ is a 2-additive capacity such that $\{P, I\}$ is representable by a Choquet integral w.r.t. $\mu$.

1. If $x_{1}, x_{2}, \ldots, x_{p} \in \mathcal{B}$ such that $\left(x_{1}, x_{2}, \ldots, x_{p}\right)$ is a strict cycle of $(P \cup I \cup M)$ then $\exists i \in\{1, \ldots, p\}: x_{i} P x_{i+1}$ (with $\left.x_{p+1}=x_{1}\right)$. This implies $\mu\left(\phi\left(x_{i}\right)\right)>$ $\mu\left(\phi\left(x_{i+1}\right)\right)$. By applying Proposition 1 , we have $\mu\left(\phi\left(x_{1}\right)\right)=\mu\left(\phi\left(x_{2}\right)\right)=$ $\ldots=\mu\left(\phi\left(x_{p}\right)\right)$, a contradiction.

2. Let $i, j, k \in N, i$ fixed such that the following system is satisfied:

$\left\{\begin{array}{l}a_{i j} \sim a_{i \vee j} \\ a_{i k} \sim a_{i \vee k} \\ i \vee j \neq i \vee k\end{array}\right.$

If there exists $l \in\{i, j, k\} \backslash\{i \vee k, i \vee j\}$ such that $a_{l} T C_{P} a_{0}$ then we have $\mu\left(\phi\left(a_{l}\right)\right)>0, \mu\left(\phi\left(a_{i j}\right)\right)=\mu\left(\phi\left(a_{i \vee j}\right)\right)$ and $\mu\left(\phi\left(a_{i k}\right)\right)=\mu\left(\phi\left(a_{i \vee k}\right)\right)$, by using Proposition 1 . Hence the monotonicity constraint of a 2-additive capacity for $\{i, j, k\}$, with $i$ fixed, $\mu_{i j}+\mu_{i k} \geq \mu_{i \vee j}+\mu_{i \vee k}+\mu_{l}$ becomes $0 \geq \mu_{l}>0$, a contradiction.

The proof of Theorem 1 is a consequence of Corollary 4 and Proposition 8 .

5.4 Proof of Corollary 1

Remark 4 Let us suppose $I=\emptyset$. Let $\left\{\mathcal{B}_{0}, \mathcal{B}_{1}, \ldots, \mathcal{B}_{m}\right\}$ be the partition of $\mathcal{B}$ built in Section 5.2. 
1. $I=\emptyset \Rightarrow \forall x, y \in \mathcal{B}, \operatorname{not}(x \sim y)$ by the definition of $\sim$. Thus any subset $K$ of $N$ such that $|K|=3$ is obviously MOPI. Hence if $(P \cup I \cup M)$ contains no strict cycle then the two conditions of Theorem 1 are satisfied.

2. $\left[a_{0}\right]=\mathcal{B}_{0}=\left\{a_{0}\right\}$ because $N B A$ is empty.

3. $\forall i, j \in N, a_{i j} \in \mathcal{B}_{s}, a_{i} \in \mathcal{B}_{r}, a_{j} \in \mathcal{B}_{t} \Rightarrow r, t<s$ with $s, r, t \in\{1, \ldots, m\}$ by applying Lemma 6 and by the defintion of the sets $\left\{\mathcal{B}_{0}, \mathcal{B}_{1}, \ldots, \mathcal{B}_{m}\right\}$.

The first part of Corollary 1 is a consequence of Theorem 1 and Remark 4 . For the second part of this corollary, by using the definition of the 2-additive capacity $\nu$ built in the proof of Theorem 1 (precisely in the proof of Proposition 7), we have $\forall i, j \in N$ such that $a_{i j} \in \mathcal{B}_{s}, a_{i} \in \mathcal{B}_{r}$ and $a_{j} \in \mathcal{B}_{t}, \mu_{i j}=$ $(2 n)^{s} \geq(n+n)(2 n)^{\max (r, t)} \geq \mu_{i}+\mu_{j}$ because $r, t<s$ (see Remark 4). Hence $I_{i j}=\mu_{i j}-\mu_{i}-\mu_{j} \geq 0$.

\subsection{Proof of Theorem 2}

Proof Let $m$ be the Möbius transform of $\mu: 2^{N} \rightarrow[0,1]$ and $k$ be an integer such that $k \geq 2$.

1. $\quad-\mu k$-monotone $\Rightarrow \forall i, j \in N, i \neq j, m_{i j} \geq 0$ (for $A=B=L$ and $|A|=2$ in the equation (15));

- if $\mu$ is monotone then $\mu_{i}=m_{i} \geq 0$;

- furthermore, using the definition of a 2-additive belief function, we have: $\mu$ is 2 -additive $\Rightarrow \mu$ is a 2 -additive belief function.

2. Let us suppose $\mu$ monotone et $k$-monotone and the existence of $i, j, k \in N$, $i$ fixed such that :

$\left\{\begin{array}{l}\mu_{i j}=\mu_{i \vee j} \\ \mu_{i k}=\mu_{i \vee k} \\ i \vee j \neq i \vee k\end{array}\right.$

and there exists $l \in\{i, j, k\} \backslash\{i \vee k, i \vee j\} \mid \mu_{l}>0$.

Then we have

$\left\{\begin{array}{l}m_{i j}+m_{i}+m_{j}=m_{i \vee j} \\ m_{i k}+m_{i}+m_{k}=m_{i \vee k} \\ i \vee j \neq i \vee k\end{array}\right.$

and there exists $l \in\{i, j, k\} \backslash\{i \vee k, i \vee j\} \mid m_{l}>0$.

In this case we have this equality:

$$
m_{i j}+m_{i k}+2 m_{i}+m_{j}+m_{k}=m_{i \vee j}+m_{i \vee k}
$$

Because $i \vee j \in\{i, j\}, i \vee k \in\{i, k\}, i \vee j \neq i \vee k$ and $l \in\{i, j, k\} \backslash\{i \vee k, i \vee j\}$, we have

$$
m_{i \vee j}+m_{i \vee k}+m_{l}=m_{i}+m_{j}+m_{k}
$$

Therefore equation (24) becomes

$$
m_{i j}+m_{i k}+m_{i}+m_{l}=0
$$

which leads to a contradiction with $m_{l}>0$. 


\subsection{Proof of Proposition 3}

Proof Let $\{P, I\}$ be an ordinal information on $\mathcal{B}$. Let us suppose that there exists $i, j, k \in N, i$ fixed such that the property $(\{i, j, k\}, i)$-MOPI is violated i.e.

$$
\left\{\begin{array}{l}
a_{i j} \sim a_{i \vee j} \\
a_{i k} \sim a_{i \vee k} \\
i \vee j \neq i \vee k
\end{array}\right.
$$

and there exists $l \in\{i, j, k\} \backslash\{i \vee k, i \vee j\} \mid a_{l} T C_{P} a_{0}$. If there exists a belief function $\mathrm{Bel}$ which represents $\{P, I\}$, we will have:

$$
\left\{\begin{array}{l}
\text { Bel }_{i j}=\text { Bel }_{i \vee j} \\
\text { Bel }_{i k}=\text { Bel }_{i \vee k} \\
i \vee j \neq i \vee k
\end{array}\right.
$$

and there exists $l \in\{i, j, k\} \backslash\{i \vee k, i \vee j\} \mid B e l_{l}>0$.

By replacing $\mu$ by Bel in the second part of the proof of Theorem 2 (see Section 5.5) and using the same reasoning, we obtain a contradiction.

\subsection{Proof of Proposition 4}

Proof Let $i, j, k \in N, i$ fixed such that

$$
\left\{\begin{array}{l}
a_{i j} \sim a_{i \vee j} \\
a_{i k} \sim a_{i \vee k} \\
i \vee j \neq i \vee k
\end{array}\right.
$$

Let be $l$ an element of $\{i, j, k\} \backslash\{i \vee k, i \vee j\}$.

1. If $l \in\{j, k\}$ then $[i \vee j=i$ if $l=j]$ or $[i \vee k=i$ if $l=k]$. So we have $a_{i l} \sim a_{i}$. Hence $\operatorname{not}\left(a_{l} T C_{P} a_{0}\right)$ because $\{P, I\}$ satisfies 2-MOPI.

2. If $l=i$ then $i \vee j=j$ and $i \vee k=k$. So $a_{i j} \sim a_{j}$ and $a_{i k} \sim a_{k}$. Therefore we have $\operatorname{not}\left(a_{l} T C_{P} a_{0}\right)$ because $\{P, I\}$ satisfies 2-MOPI.

\subsection{Proof of Theorem 3}

Proof Let $\{P, I\}$ an ordinal information.

1. $(\Rightarrow)$ We suppose that $\{P, I\}$ is representable by a 2 -additive belief function Bel.

(a) It is easy to show that $(P \cup I \cup M)$ contains no strict cycle;

(b) If there exists $i, j \in N, i \neq j$ such that $a_{i j} \sim a_{j}$ and $a_{i} T C_{P} a_{0}$ then $m_{i j}<0$ with $m$ the mass distribution of $\mathrm{Bel}$. That is impossible because Bel is a belief function.

2. $(\Leftarrow)$ Let us suppose that

- $(P \cup I \cup M)$ contains no strict cycle;

- $\{P, I\}$ satisfies the $2-M O P I$ property. 
Using the Proposition 4, the conditions of Theorem 1 are satisfied. Let $\left\{\mathcal{B}_{0}, \mathcal{B}_{1}, \ldots, \mathcal{B}_{m}\right\}$ be a partition of $\mathcal{B}$ built like in Section 5.2. Let us define the mapping $f: \mathcal{B} \rightarrow \mathbb{R}$ and $\mathrm{Bel}: 2^{N} \rightarrow[0,1]$ as follows: For $i \in\{0, \ldots, m\}$,

$$
\begin{gathered}
\forall x \in \mathcal{B}_{i}, f(\phi(x))= \begin{cases}0 & \text { if } i=0 \\
(2 n)^{i} & \text { otherwise. }\end{cases} \\
\left\{\begin{array}{l}
\operatorname{Bel}_{\emptyset}=0 \\
B e l_{i}=\frac{f_{i}}{\alpha}, \forall i \in N \\
B e l_{i j}=\frac{f_{i j}}{\alpha}, \forall i, j \in N \\
B e l(K)=\sum_{\{i, j\} \subseteq K} f_{i j}-(|K|-2) \sum_{i \in K} B e l_{i}, \forall K \subseteq N,|K|>2 .
\end{array}\right.
\end{gathered}
$$

where $\alpha=\sum_{\{i, j\} \subseteq N} f_{i j}-(n-2) \sum_{i \in N} f_{i}$

$\mathrm{Bel}$ define like this is 2-additive (the proof is similar to the proof of Theorem 1 in Section 5.3). The last thing we have to prove is $\forall i, j \in N, i \neq j$, $m_{i j}=B e l_{i j}-\mathrm{Bel}_{i}-\mathrm{Bel}_{j} \geq 0$ where $m$ is the mass distribution of Bel. Let $i, j \in N, i \neq j$.

- If $a_{i j} \sim a_{i}$ then $m_{i j}=0$ because $\{P, I\}$ satisfies the 2-MOPI property. Using the same reasons if $a_{i j} \sim a_{j}$ then $a_{j i} \sim a_{j}$ and $m_{i j}=0$;

- If $a_{i j} T C_{P} a_{i}$ and $a_{i j} T C_{P} a_{j}$ then there exists $p, q, s \in\{0, \ldots, m\}$ such that $a_{i j} \in \mathcal{B}_{p}, a_{i} \in \mathcal{B}_{q}, a_{j} \in \mathcal{B}_{s}$ with $p>q$ and $p>s$. So we have $f_{i j}=(2 n)^{p}, f_{i}=(2 n)^{q}, f_{j}=(2 n)^{s}$ and then Bel $_{i j} \geq B e l_{i}+B e l_{j}$.

\section{References}

C. A. Bana e Costa, F. Nunes da Silva, and J.-C. Vansnick. Conflict dissolution in the public sector: A case-study. European Journal of Operational Research, 130:388-401, 2001.

C. A. Bana e Costa, E. C. Correa, J.-M. De Corte, and J.-C. Vansnick. Facilitating bid evaluation in public call for tenders: A socio-technical approach. Omega, 30:227-242, 2002.

C. A. Bana e Costa, J.-M. De Corte, and J.-C. Vansnick. On the mathematical foundations of MACBETH. Multiple Criteria Decision Analysis: State of the Art Surveys, 78:409-437, 2005.

L. Berrah and V. Clivillé. Towards an aggregation performance measurement system model in a supply chain context. Computers in Industry, 58(7): 709-719, 2007.

A. Chateauneuf. Modeling attitudes towards uncertainty and risk through the use of Choquet integral. Annals of Operations Research, 52:3-20, 1994.

A. Chateauneuf. Ellsberg paradox intuition and Choquet expected utility. In G. Coletti, D. Dubois, and R. Scozzafava, editors, Mathematical models for handling partial knowledge in artificial intelligence, pages 1-20. Plenum Press, 1995. 
A. Chateauneuf and J.Y. Jaffray. Some characterizations of lower probabilities and other monotone capacities through the use of Möbius inversion. Mathematical Social Sciences, 17:263-283, 1989.

A. Chateauneuf, R. Kast, and A. Lapied. Conditioning capacities and choquet integrals: The role of comonotony. Theory and Decision, 51(2-4):367-386, 2004.

A. Chateauneuf, M. Grabisch, and A. Rico. Modeling attitudes towards uncertainty through the use of Sugeno integral. J. of Mathematical Economics, 44:1084-1099, 2008.

G. Choquet. Theory of capacities. Annales de l'Institut Fourier, 5:131-295, 1953.

V. Clivillé, L. Berrah, and G. Mauris. Quantitative expression and aggregation of performance measurements based on the MACBETH multi-criteria method. International Journal of Production economics, 105:171-189, 2007.

D. Ellsberg. Risk, ambiguity, and the Savage axioms. Quart. J. Econom., 75: 643-669, 1961.

T. Gajdos. Measuring inequalities without linearity in envy: Choquet integrals for symmetric capacities. J. of Economic Theory, 106:190-200, 2002.

M. Grabisch. The application of fuzzy integrals in multicriteria decision making. European J. of Operational Research, 89:445-456, 1996.

M. Grabisch. $k$-order additive discrete fuzzy measures and their representation. Fuzzy Sets and Systems, 92:167-189, 1997.

M. Grabisch and C. Labreuche. Fuzzy measures and integrals in MCDA. In J. Figueira, S. Greco, and M. Ehrgott, editors, Multiple Criteria Decision Analysis, pages 563-608. Kluwer Academic Publishers, 2004.

M. Grabisch and Ch. Labreuche. A decade of application of the Choquet and Sugeno integrals in multi-criteria decision aid. 4OR, 6:1-44, 2008.

M. Grabisch, J. Duchêne, F. Lino, and P. Perny. Subjective evaluation of discomfort in sitting position. Fuzzy Optimization and Decision Making, 1 (3):287-312, 2002.

M. Grabisch, Ch. Labreuche, and J.-C. Vansnick. On the extension of pseudoBoolean functions for the aggregation of interacting bipolar criteria. Eur. J. of Operational Research, 148:28-47, 2003.

C. K. Hsee. The evaluability hypothesis: An explanation for preference reversals between joint and separate evaluations of alternatives. Organizational Behavior and Human Decision Processes, 67:242-257, 1996.

J.-Y. Jaffray. Linear utility theory for belief functions. Operations Research Letters, 8:107-112, 1989.

J.-Y. Jaffray and P. Wakker. Decision making with belief functions: Compatibility and incompatibility with the sure-thing principle. Journal of Risk and Uncertainty, 7:255-271, 1993.

Ch. Labreuche and M. Grabisch. The Choquet integral for the aggregation of interval scales in multicriteria decision making. Fuzzy Sets and Systems, 137:11-26, 2003.

T. Marchant. Towards a theory of MCDM: Stepping away from social choice theory. Mathematical Social Sciences, 45(3):343-363, 2003. 
B. Mayag, M. Grabisch, and C. Labreuche. A characterization of the 2-additive Choquet integral. In Proceedings of IPMU'08 (CD), Malaga, Spain, June 2008.

P. Miranda, M. Grabisch, and P. Gil. p-symmetric fuzzy measures. Int. J. of Uncertainty, Fuzziness, and Knowledge-Based Systems, 10 (Suppl.):105123, 2002.

P. Miranda, M. Grabisch, and P. Gil. Axiomatic structure of $k$-additive capacities. Mathematical Social Sciences, 49:153-178, 2005.

T. Murofushi and S. Soneda. Techniques for reading fuzzy measures (III): interaction index. In 9th Fuzzy System Symposium, pages 693-696, Sapporo, Japan, May 1993. In Japanese.

E. Pap. Null-Additive Set Functions. Kluwer Academic, 1995.

J.P. Pignon and Ch. Labreuche. A methodological approach for operational and technical experimentation based evaluation of systems of systems architectures. In Int. Conference on Software $\& 3$ Systems Engineering and their Applications (ICSSEA), Paris, France, December 4-6 2007.

D. Schmeidler. Integral representation without additivity. Proc. of the Amer. Math. Soc., 97(2):255-261, 1986.

D. Schmeidler. Subjective probability and expected utility without additivity. Econometrica, 57(3):571-587, 1989.

G. Shafer. A Mathematical Theory of Evidence. Princeton Univ. Press, 1976.

L. S. Shapley. A value for $n$-person games. In H. W. Kuhn and A. W. Tucker, editors, Contributions to the Theory of Games, Vol. II, number 28 in Annals of Mathematics Studies, pages 307-317. Princeton University Press, 1953.

H. Simon. Rational choice and the structure of the environment. Psychological Review, 63(2):129-138, 1956.

P. Slovic, M. Finucane, E. Peters, and D. G. MacGregor. The affect heuristic. In T. Gilovitch, D. Griffin, and D. Kahneman, editors, Heuristics and biases: the psychology of intuitive judgment, pages 397-420. Cambridge University Press, 2002.

Ph. Smets. Decision making in the tbm: the necessity of the pignistic transformation. Int. J. Approx. Reasoning, 38(2):133-147, 2005.

A. Tversky and D. Kahneman. Advances in prospect theory: cumulative representation of uncertainty. J. of Risk and Uncertainty, 5:297-323, 1992.

J. Von Neuman and O. Morgenstern. Theory of Games and Economic Behavior. Princeton University Press, 1944.

S. Weber. $\perp$-decomposable measures and integrals for Archimedean t-conorms L. J. Math. Anal. Appl., 101:114-138, 1984.

J. A. Weymark. Generalized Gini inequality indices. Mathematical Social Sciences, 1:409-430, 1981. 